\title{
Wearable Devices Downlink and Uplink Transmission in Multi-hop HetNet with Full-Duplex Relay
}

\begin{abstract}
A Thesis Submitted to the Department of Computer Science and Communications Engineering, the Graduate School of Fundamental Science and Engineering of Waseda University in Partial Fulfillment of the Requirements for the Degree of Master of Engineering
\end{abstract}

July $24^{\text {th }}, 2018$

Rong Ye

(5116FG25-5)

Advisor: Prof. Shigeru Shimamoto

Research guidance: Research on Wireless Access 


\section{Table of Contents}

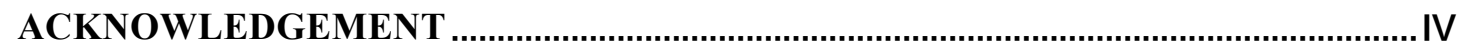

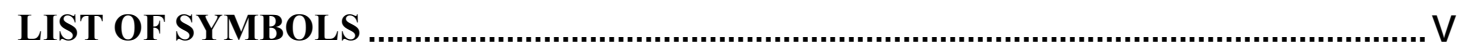

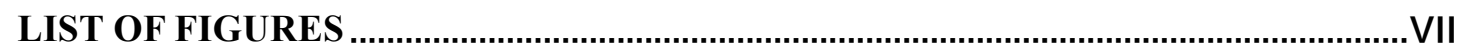

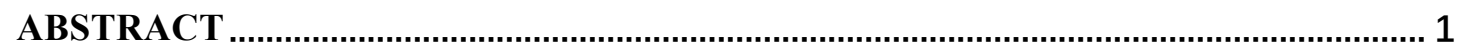

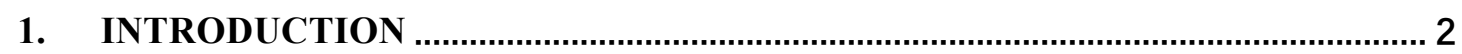

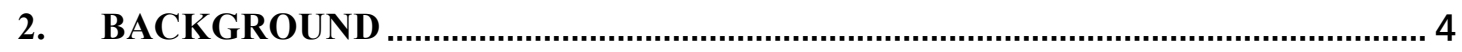

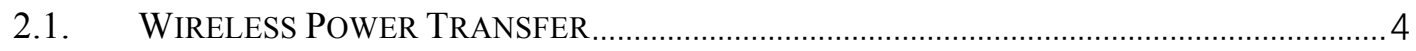

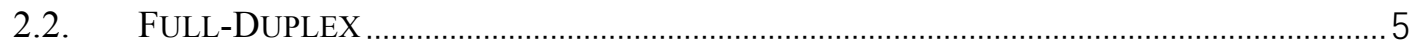

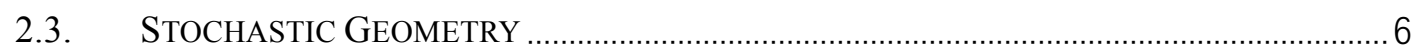

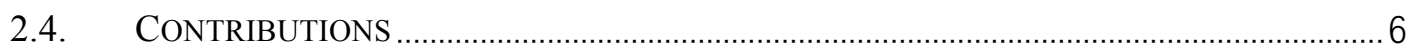

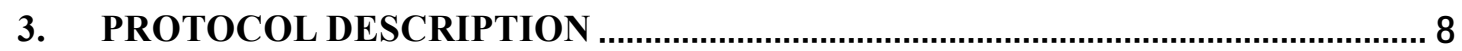

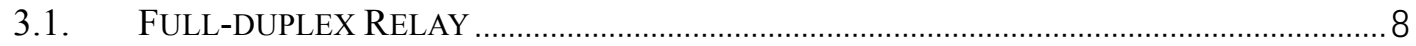

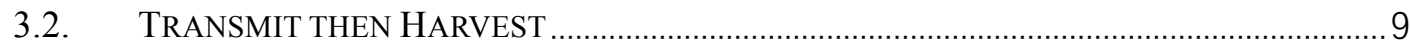

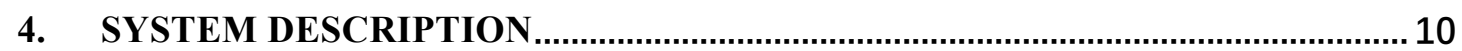

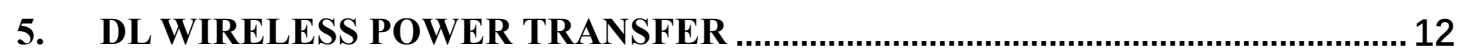

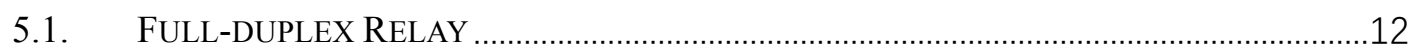

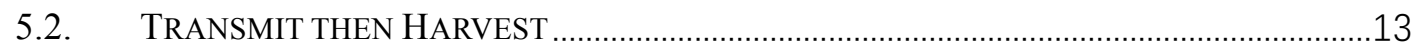

6. UL INFORMATION TRANSMISSION ............................................................ 15

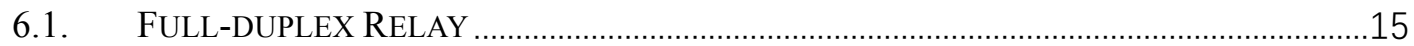

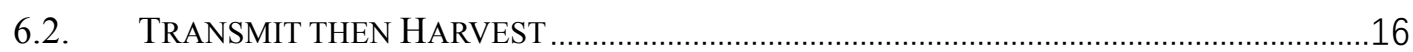

7. EXACT ANALYSIS OF DL AND UL TRANSMISSION ........................................ 17

7.1. PRoBabILITY DENSITY FUNCTION OF DiSTANCE_........................................................17

7.2. PHASE-1 DL WIRELESS POWER TRANSFER ………………………………...................17

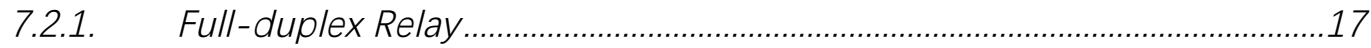

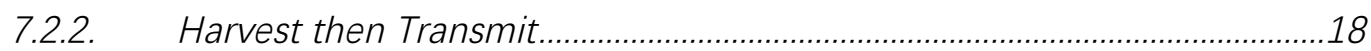

7.3. PHASE-2 UL INFORMATION TRANSMISSION ………………………………………….....19

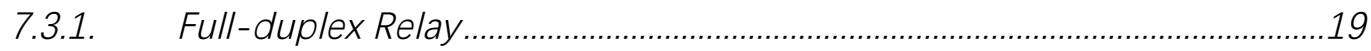

7.3.2. Harvest then Transmit........................................................................................ 


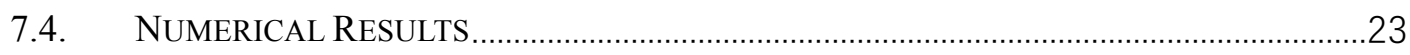

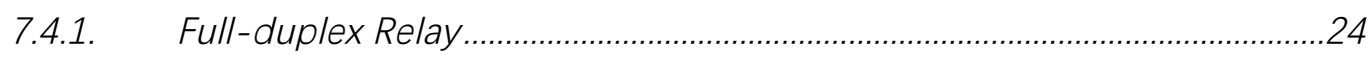

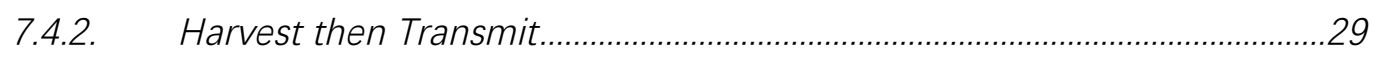

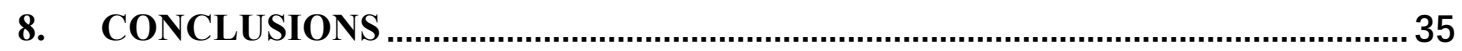

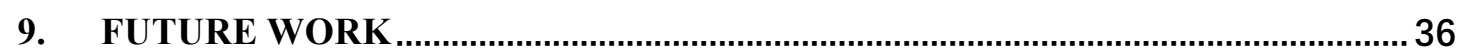

APPENDIX

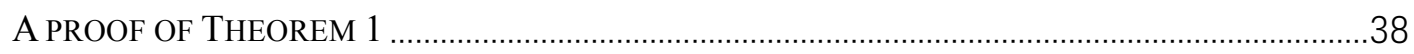

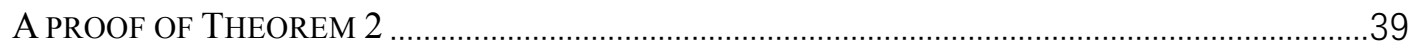

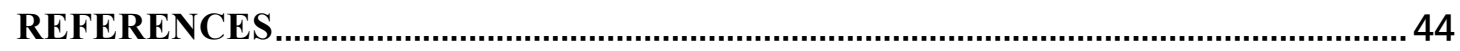

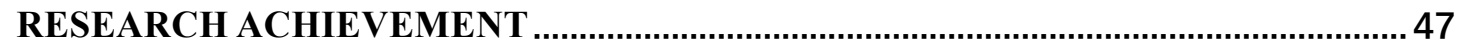




\section{Acknowledgement}

I would like to thank my advisor, Prof. Shigeru Shimamoto firstly for the 2 years of guidance, support, encouragement and patient has given me. I feel incredibly fortunate to have him as my advisor and have an opportunity to study in Shimamoto Lab. Prof. Shigeru Shimamoto taught me how to do the research on wireless communication, discuss the research direction with me and always give me the important suggestion on my research problems with patient. Thought all the efforts I have paid for my research paper, it would not possible to give a presentation on an international conference without his support. It is my honor to be one of his students and the things that he inspired me not only for research and for life and work also.

I appreciate all the help from the LTE group members Dr. Zhenni Pan, Dr. Kang Kang, Huan Wang and Mulatu Baisa Taye, it would not possible to complete my research without the good advice from them, they always inspired me.

I am also thanks to my friends Yanan Gao and Rakotoaritina Tojoarisoa for the support and patient from the bottom of my heart.

Thanks to my family, parents and grandparents, they helped me to have the chance to study in Japan for my master program. 


\section{List of Symbols}

\section{Symbol/Subscript}

$E_{U m}$

$E_{U S}$

$E_{U S}^{\prime}$

$\Phi_{B S}, \lambda_{B S}$

$\Phi_{U m}, \lambda_{U m}$

$\Phi_{U s}, \lambda_{U s}$

$\tau, T$

$\eta$

$\beta$

$P_{B S}$

$P_{U m}$

$h$

$g$

$L_{o}(\cdot)$

$|X|$

$\alpha_{U}$

$\alpha_{B}$

I

$d$

$d_{0}$

SINR $_{U m}$

SINR $_{U m}^{\prime}$

$$
\sigma^{2}
$$

$R_{U m}^{\text {Exact }}$

\section{Description}

Total harvested energy on tier 1 full-duplex relay

Total harvested energy on tier 2 WTDs in the situation of tier 1 users are full-duplex relay

Total harvested energy on tier 2 WTDs in the situation of tier 1 users are harvest then transmit relay

Picocell PPP and density

Tier 1 relay users and density

Tier 2 WTDs and density

Time slot factor and time slot

RF to DC transform efficiency

The frequency dependent constant value

Transmit power of picocell BSs on DL

Transmit power of tier 1 relay users on DL

Small scale fading channel gain

Small scale fading channel gain

Path loss function

The distance between the picocell BS and the 2-tier users

Path loss exponent between the 2-tier users

Path loss exponent between the picocell BSs and 2-tier users

Interference from the ambient RF

Reference distance

Constant distance value between the tier 1 relay users and the tier 2 WTDs

SINR received on the tier 1 full-duplex relay

SINR received on the tier 1 harvest then transmit relay

Noise

Average ergodic rate received on tier 1 full-duplex relay 


$\begin{array}{cl}R_{U m}^{\prime \text { Exact }} & \text { Average ergodic rate received on tier } 1 \text { harvest then } \\ & \text { transmit relay } \\ R_{1} & \text { SINR threshold } \\ P_{\text {out }}^{U L} t_{U m} & \text { UL Outage probability with tier } 1 \text { full-duplex relay } \\ P_{\text {out }_{U m}}^{\prime L} & \text { UL Outage probability with tier } 1 \text { harvest then transmit } \\ & \text { relay } \\ \mathcal{L}(\cdot) & \text { Laplace function } \\ \exp (\cdot) & \text { Exponential distribution function } \\ \mathbb{E}(\cdot) & \text { Expectation operator }\end{array}$




\section{List of Figures}

Fig. 1. Heterogeneous network model 3

Fig. 2. Model for an energy harvesting device 4

Fig. 3. Model for a full-duplex smart phone relay 5

Fig. 4. Voronoi tessellation of the locations 6

Fig. 5. The full-duplex relay protocol 8

Fig. 6. The harvest then transmit relay protocol 9

Fig. 7. The system network models 10

Fig. 8. The Average Harvested Energy on Tier 2 User with full-duplex relay 24

Fig. 9. The Average Harvested Energy on Tier 1 User with full-duplex relay 25

Fig. 10. The Average Harvested Energy on 2-Tier User with full-duplex relay. 26

Fig. 11. The Average Harvested Energy on Tier 2 User with full-duplex relay 26

Fig. 12. The Average Ergodic Rate of tier 2 WTD on UL with full-duplex relay... 27

Fig. 13. The UL outage probability of tier 2 WTD with full-duplex relay.... 28

Fig. 14. The UL outage probability of tier 2 WTD with full-duplex relay. 29

Fig. 15. The Average Harvested Energy on Tier 2 User with harvest then transmit relay 29

Fig. 16. The comparison of Average Harvested Energy between the the full-duplex relay and harvest then transmit relay with transmit power. 30

Fig. 17. The comparison of Average Harvested Energy between the full-duplex relay and harvest then transmit relay with RF to DC transform efficiency. 
Fig. 18. The comparison of Average Ergodic Rate between the full-duplex relay and harvest then transmit relay with time slot 32

Fig. 19. The comparison of Outage Probability between the full-duplex relay and harvest then transmit relay with path loss exponent 33

Fig. 20. The comparison of Outage Probability between the full-duplex relay and harvest then transmit relay with path loss exponent 34

Fig. 21. The 3-D system model based on HPPP 37 


\section{Abstract}

This paper we provide a tractable system for 2-tier users, wearable device terminals (WTDs) that in tier 2 and under the control of tier 1 relay users. Tier 1 user works as two kinds of relay, full-duplex relay and the harvest then transmit relay to charge the WTDs on the downlink (DL) directly based on the technology of wireless power transfer (WPT), then helps WTDs to transmit the information on the uplink (UL). 2-tier of users and picocell base stations (BS) are randomly distributed based on homogeneous poison point process (HPPP). We only considered the user association between the tier 1 users and the picocell BSs that tier 1 users always connected to the closest BS on UL since WTDs tier 2 users are close enough to tier 1 users. We analyze the processes of wireless power transfer on DL and information transmission on UL. We will derive the analysis of the average harvested energy on DL for both 2-tier users, the average ergodic rate and outage probability on UL for WTDs tier 2 users. According to the analytical results, the performance of the situation of tier 1 users are the fullduplex relay is better compare to the situation of tier 1 users are the harvest then transmit relay, it is observed that with the increasing of the density of the picocell base stations and the allocated time slot for energy harvesting, the average harvested energy will improve due to the decreasing of the UL performance, the average ergodic rate and UL outage probability will degrade significantly. 


\section{Introduction}

As the WTDs developed rapidly, the WTDs played the important role in our day life, wearable communication networks will be the next frontier type of networks for wireless communications. A main challenge for wearable networks is to find green methods to support them in dense environments with lower latency, higher energy efficiency [1], [2], [3]. As we know that traditional energy harvesting sources such as solar, wind, hydroelectric depend on the locations and environments. Wireless power transfer (WPT) is an alternative approach to prolong the lifetime of mobile devices since the radio signals carry the information and the energy at the same time [4]. With the deployment of heterogeneous network (HetNet) in Fig.1, lower transmit power BSs such as picocells, fetmocells and remote radio heads are used to extend the edge coverage in cellular networks ranging from an open environment to office buildings, homes, subway station and airport where the places with high visitor flowrate [5], [9]. As long with the higher density distribution of WTDs (such as smart glasses, smart watches, smart shoes, etc.) in these kinds of scenario, it is urgently required us to pay attention on how to prolong the lifetime for them. An analytical model for k-tier MIMO applied HetNet has been proposed, the different user association scheme can make sure the users harvest more energy on DL and minimized the effect of path loss on UL [6]. In addition, the potentially harmful interference received by the users will become a precious new source of energy. Recently, the potential of harvesting the ambient RF in fifth-generation (5G) networks has been investigated [7], [8]. Moreover, full-duplex 
(FD) communication is capable to increase the capacity and double the spectral efficiency through transmitting and receiving on the same channel at the same time, but it will suffer from the self-interference that the receiver will be impacted by the transmitter. Along with the achievement in signal designs, mitigation schemes and selfinterference cancellation methods [10], full-duplex can potentially be utilized in heterogeneous cellular network [11].

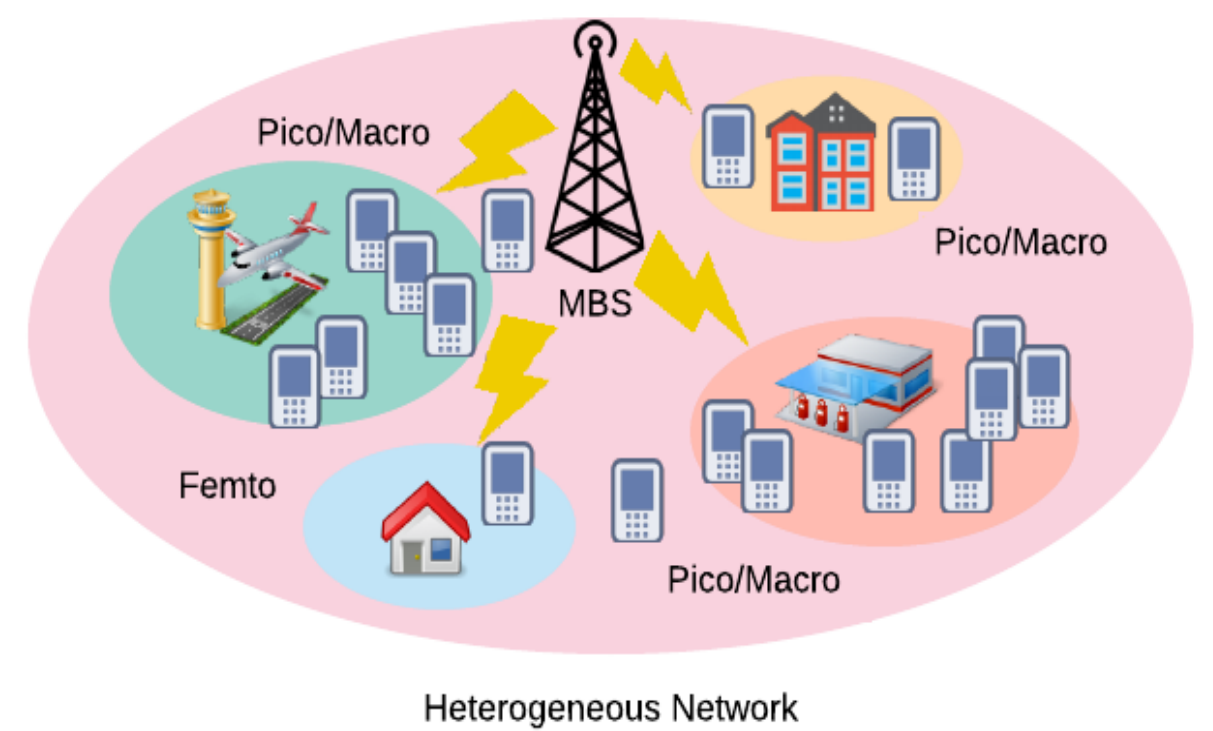

Fig. 1. Heterogeneous network model 


\section{Background}

\subsection{Wireless Power Transfer}

Unlike inductive coupling and magnetic resonance coupling, radio frequency has no limitation of transfer distance, so the harvested power can be transformed into direct current by using of the wireless energy transfer technique. For improving the energy efficiency, WPT is the green method that can be used in HetNet, cellular network, D2D and sensor communication networks to charge the devices. WPT can be divided into two kinds of protocols, harvest than transmit and simultaneous wireless information and power transfer [12]. Fig.2 is a model for an energy harvesting device that consists of an antenna, energy harvesting unit, energy storage unit and an information transmission unit.

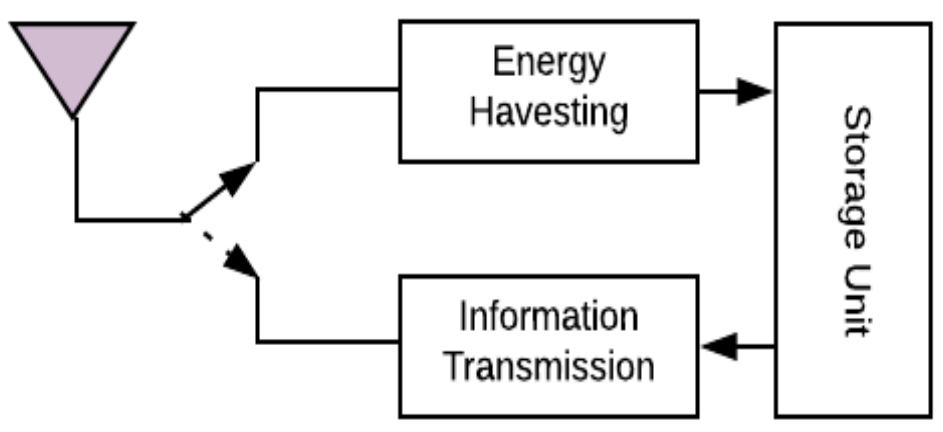

Fig. 2. Model for an energy harvesting device 


\subsection{Full-Duplex}

Half-duplex (HD) is widely used in traditional cellular network (e.g. TDD, FDD), FD communication is the advance way to double the capacity of cellular network compare to HD, but it will suffer from the self-interference between the receive antenna and transmit antenna. Fig. 3 is the model of smartphone FD relay that the help the WTDs to transmit the information on both DL and UL, BS and the WTDs are TDMA communication and WTDs do not have to connect the BS directly.

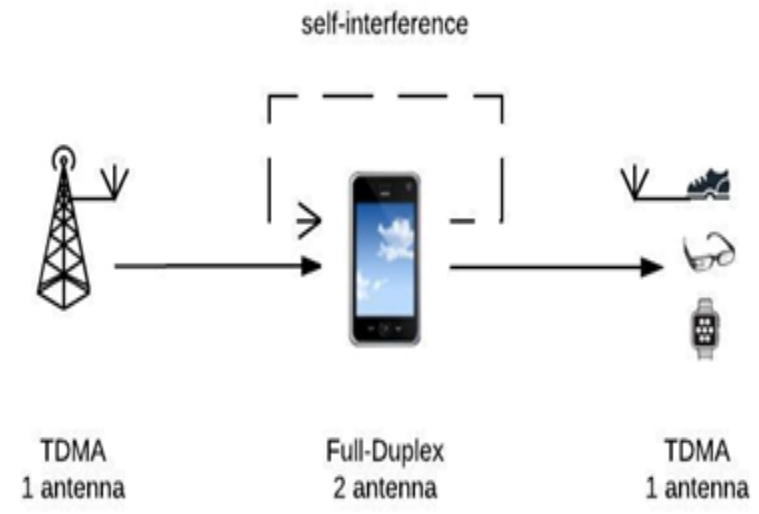

Fig. 3. Model for a full-duplex smart phone relay 


\subsection{Stochastic Geometry}

Stochastic geometry is traditionally used in ecology and geology area, but it also can randomly model the points, objects etc in a complicated given place. Especially in HetNet, it is very useful method to help us analyze the position of each points. Poisson Point Process (PPP) is the enabling method to resolve the problem of wireless communication system because the uncertainty location of the users.

Voronoi tessellation is a kind of simulation method to model the location of the BSs and 2-tier users with their own density based on the Homogeneous Poisson Point Process (HPPP). Firstly, simulate 19 picocells, then point the tier 1 relay users based on the random distance from the picocell BSs. Finally, point the WTDs based on the distance from the tier 1 relay users. The triangles mean the location of picocell BSs, blue circles mean the tier 1 relay users and the red stars mean the tier 2 WTDs.

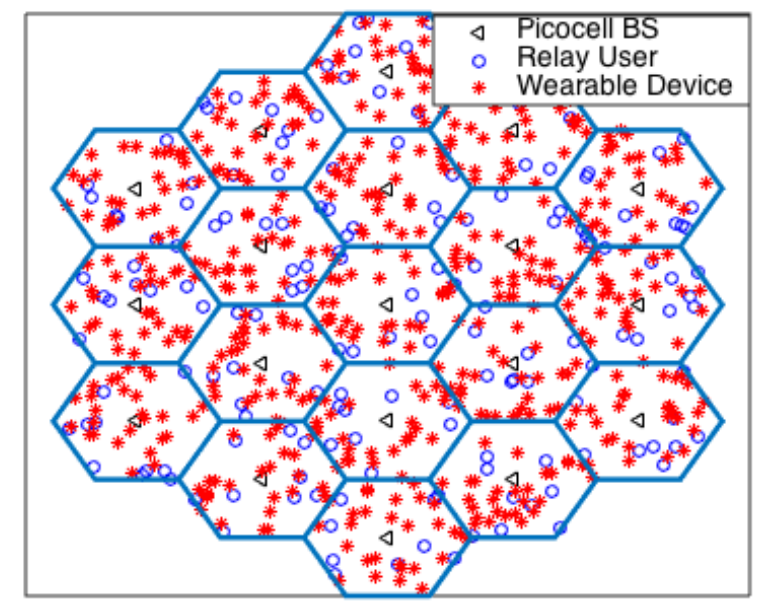

Fig. 4. Voronoi tessellation of the locations

\subsection{Contributions}

We present a tractable system model that all the WTDs are central under controlled 
by high level priority relay terminal devices such as smart phones, pad. Especially some low power devices such like smart watches, smart glasses, wearable fitness trackers, accessories, etc. Imagining that many people may bring more than 3 kinds of WTDs, it is inconvenient to charge them in the crowded places which with a high visitor flowrate. Unlike [13], we propose that the picocell BS and 2-ter users randomly located with particular transmit power, path loss exponent and density.

We compare the situation of tier 1 users are full-duplex relay and the situation of tier 1 users are the harvest then transmit relay. Firstly, we derive the analysis of the average harvested energy DL for both 2-tier users, that shows how much energy the users can harvest from the DL and ambient RF at the same time by the two kinds of relay methods. We analyze the comparison of outage probability and the average ergodic rate on UL for WTDs tier 2 users. The outage probability relate to the SINR threshold equals to the CDF of the SINR. The average ergodic rate is integrated by the throughput threshold. 


\section{Protocol Description}

\subsection{Full-duplex Relay}

The time slot can be divided into two parts, $\tau T$, and $(1-\tau) T$, where $\tau, \tau \in(0,1)$ is the time allocation factor. In terms of tier 1 users are the full-duplex relays, $\tau T$ is the time slot for tier 1 relay users and the tier 2 WTDs to harvest the energy on DL from the previous tier directly and can also harvest from the ambient radio frequency. On the UL, the 2 tier users can use the harvest energy as the transmit power to transmit the information on the rest of the time slot $(1-\tau) T$.

\begin{tabular}{|c|c|}
\hline & \\
\hline EH: BS to Tier1 TD & IT: Tier1 TD to BS \\
\hline EH: Tier1 TD to Tier2 TD & IT: Tier2 TD to Tier1 TD \\
\hline $\begin{array}{c}\text { Energy harvesting } \\
\text { process }\end{array}$ & $\begin{array}{c}\text { Information transmission } \\
\text { process }\end{array}$ \\
\hline
\end{tabular}

Fig. 5. The full-duplex relay protocol 


\subsection{Transmit then Harvest}

The time slot can be divided into two parts, $\tau T$, and $(1-\tau) T$, where $\tau, \tau \in(0,1)$ is the time allocation factor. $\tau T$ is the time slot for WTDs to harvest the energy on DL from the tier 1 relay users and RF, the rest of the time slot $(1-\tau) T$ can be divided into two parts, $\frac{(1-\tau) T}{2}$ is the time slot for WTDs users to use the harvested energy as transmit power to transmit the information to the tier 1 users, then the left $\frac{(1-\tau) T}{2}$ is the time slot for tier 1 relay users to upload the information to the picocell BSs on UL [14].

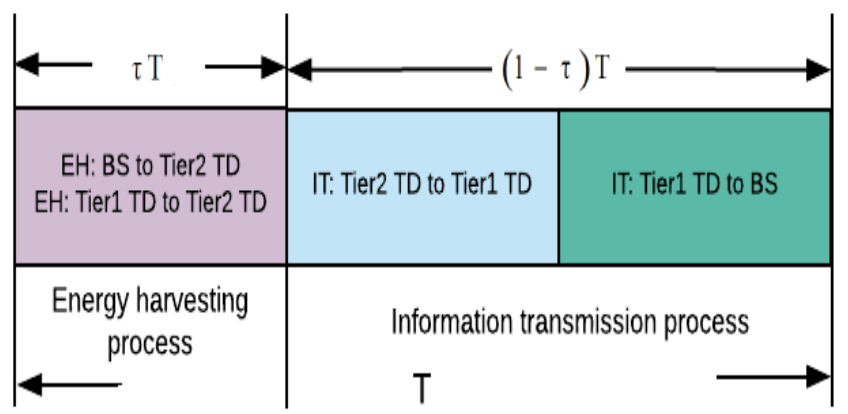

Fig. 6. The harvest then transmit relay protocol 


\section{System Description}

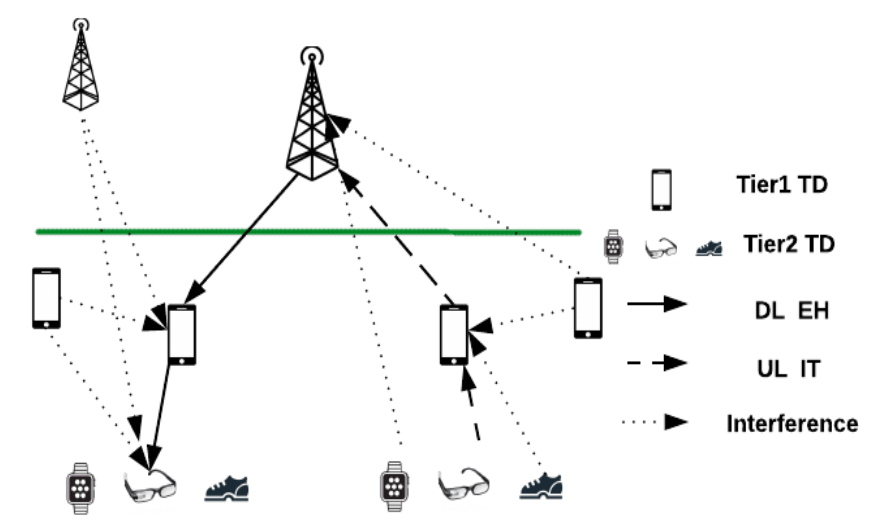

Fig. 7. The system network models

Imagine that in the situation of high visitor rate, each person may have at least 3 WTDs, it is inconvenient to charge them at the same time. In order that we propose this tractable system model to charge the low power consumption WTDs. The entire communication consists of two different phases, namely, energy harvesting phase and wireless information transfer phase. Each tier of user harvests energy from direct DL and the ambient RF.

Fig.7 depicts 2-tier users deployed in picocell network such as tier 1 relay and WTD tier 2 users. We assume that each picocell BS is equipped with single antenna. The locations of picocell BSs and 2-tier users are modeled following an independent HPPP $\Phi_{B S}$ with density $\lambda_{B s}, \lambda_{U m}, \lambda_{U s}$. In the high density HetNet, the density of the WTDs tier 2 users is much larger than picocell BSs and the tier 1 users, considering that there is only one active WTDs tier 2 user and tier 1 user at each time slot in each picocell. 
In this system model, we do not consider direct energy transfer and information transfer with WTDs tier 2 user and picocell base stations, because picocell is limited resources and only server for tier 1 users because tier 1 users have high requirement in information transfer and energy. As we know the full-duplex relay will suffer from the self-interference, but along with the development of the self-interference cancellation (SIC) to further reduce the interference by using analog/digital domain SIC, although this part of study is still challengeable. We considered the self-interference can be perfectly cancelled in this paper [10]. 


\section{DL Wireless Power Transfer}

\subsection{Full-duplex Relay}

In the picocell, only one user of each tier can be allowed to communicate with its serving picocell BS at a divided time slot, the allocate power transfer time for each tier user is $\tau T$, where $\tau \in(0,1)$. In the situation of tier 1 users are the full-duplex relay, on the phase of energy harvesting, the total harvest energy on a typical tier 1 user o that is associated with the closest picocell base station and the total harvest energy at a typical WTD tier 2 user o are given by

$$
\left\{\begin{array}{c}
E_{U m}=\eta \tau T P_{B s} g_{o} L_{0}\left(\max \left\{\left|X_{o, m}\right|, d\right\}\right)+\eta \tau T\left(I_{B s}+I_{m u}\right) \\
E_{U s}=\eta \tau T P_{U m} h_{o} L_{0}\left(\max \left\{\left|X_{o, u}\right|, d\right\}\right)+\eta \tau T\left(I_{B S}^{\prime}+I_{m u}^{\prime}\right)
\end{array},\right.
$$

where $E_{U m}, E_{U s}$ are the ideal total energy that 2-tier users can harvest on the DL from the serving picocell BS directly, also can be charged from the interfered users, $\eta$ $(0<\eta<1)$ is the RF-to-DC conversion efficiency, $P_{B S}, P_{U m}$ are the picocell transmit power and tier 1 user transmit power, respectively, $L_{0}(\max \{|X|, d\})=\beta|X|^{-\alpha}$ is the path loss function, $\beta$ is the frequency dependent constant value, $\alpha(2<\alpha<4)$ is the path loss exponent, $d$ is the reference distance, $g_{o}, h_{o} \sim \exp (1)$ and $\left|X_{o, m}\right|,\left|X_{o, u}\right|$ are the small-scale fading channel power gain, by mean 1, the distance between the serving picocell BS and the typical tier 1 user, and the distance between the tier 1 user and the active WTD tier 2 user, respectively based on the Rayleigh fading. The energy harvest from the interference can be given by 


$$
\left\{\begin{array}{rl}
I_{B s} & =\sum_{i \in \Phi_{B s} \backslash\{0\}} P_{B s} g_{b, o} L_{0}\left(\max \left\{\left|X_{i}\right|, d\right\}\right) \\
I_{m u} & =\sum P_{U m} g_{m, o} L_{0}\left(\max \left\{\left|X_{j}\right|, d\right\}\right)
\end{array},\right.
$$

and

$$
\left\{\begin{array}{l}
I_{B S}^{\prime}=\sum P_{B s} h_{b, o} L_{0}\left(\max \left\{\left|X_{k}\right|, d\right\}\right) \\
I_{m u}^{\prime}=\sum_{s \in \Phi_{U m} \backslash\{o\}} P_{U m} h_{m, o} L_{0}\left(\max \left\{\left|X_{s}\right|, d\right\}\right)
\end{array}\right.
$$

where $I_{B S}, I_{m u}, I_{B S}^{\prime}, I_{m u}^{\prime}$ are the sum of the interference harvest from the DL under the condition of full-duplex relay network, including the interference from the interfering picocell BSs and interfering tier 1 users, $g, h \sim \exp (1),|X|$ are the smallscale fading interfering channel power gain by mean 1 , the distance between interfering picocell BSs $i \in \Phi_{B S} \backslash\{o\}$ (except the serving picocell BS) or tier 1 users $s \in \Phi_{U m} \backslash$ $\{o\}$ (except the serving tier 1 user) and the typical charged users, respectively, based on the Rayleigh fading.

\subsection{Transmit then Harvest}

In the situation of tier 1 users are the harvest then transmit relay, on the phase of energy harvesting, the total harvest energy on a typical tier 2 user o that is given by

$$
E_{U S}^{\prime}=\eta \tau T P_{U m} h_{o} L_{0}\left(\max \left\{\left|X_{o, u}\right|, d\right\}\right)+\eta \tau T I_{B S}^{\prime}
$$

where $E_{U S}^{\prime}$ is the ideal total energy that tier 2 WTDs users can harvest on the DL from the tier 1 relay users directly, also can be charged from the interfered picocell BSs, $\eta$ $(0<\eta<1)$ is the RF-to-DC conversion efficiency, $P_{U m}$ is transmit power of the tier 1 relay user, $L_{0}(\max \{|X|, d\})=\beta|X|^{-\alpha}$ is the path loss function, $\beta$ is the frequency dependent constant value, $\alpha(2<\alpha<4)$ is the path loss exponent, $d$ is the reference 
distance, $h_{o} \sim \exp (1)$ and $\left|X_{o, u}\right|$ are the small-scale fading channel power gain, by mean 1 , the distance between the tier 1 relay users and the typical tier 2 WTDs users based on the Rayleigh fading. The energy harvest from the ambient RF can be given by

$$
I_{B S}^{\prime}=\sum P_{B S} h_{b, o} L_{0}\left(\max \left\{\left|X_{k}\right|, d\right\}\right)
$$

unlike the situation of tier 1 users are the full-duplex relay, the interference only comes from the interfered picocell BSs, where $I_{B S}^{\prime}$ is the sum of the interference harvest from the interfering picocell BSs, $h \sim \exp (1),\left|X_{k}\right|$ are the small-scale fading interfering channel power gain by mean 1 , the distance between interfering picocell BSs $i \in \Phi_{B S}$ and the typical charged WTDs users, respectively, based on the Rayleigh fading. 


\section{UL Information Transmission}

\subsection{Full-duplex Relay}

On UL information transmission phase, the tier 1 users and 2 tier 2 WTDs will transmit the information to the picocell BSs at the same time slot. In terms of the tier 1 users are the full-duplex relay, the 2-tier users will make use of the harvested energy as transmit power to transmit the data on UL at the time slot $(1-\tau) T$, where $\tau \in(0,1)$. SINR received on the tier 1 full-duplex user from typical WTD tier 2 user can be given as

$$
\operatorname{SINR}_{U m}=\frac{P_{U S}^{D L} h^{\prime}{ }_{o} L_{0}\left(\max \left\{\left|X_{o, u}^{\prime}\right|, d\right\}\right)}{I^{\prime}{ }_{m u}+I^{\prime}{ }_{u s}+\sigma^{2}}
$$

based on the Shannon theorem where $P_{U S}^{D L}=\frac{E_{U s}}{(1-\tau) T}, P_{U m}^{D L}=\frac{E_{U m}}{(1-\tau) T}, h^{\prime}{ }_{o} \sim \exp (1)$, $\left|X_{o, u}^{\prime}\right|$ are the small-scale fading interfering channel power gain by mean 1 , the distance between typical WTD tier 2 user and connected tier full-duplex 1 user, respectively.

In addition,

$$
\left\{\begin{aligned}
I_{m u}^{\prime} & =\sum P_{U m}^{D L} g_{m, o}^{\prime} L_{0}\left(\max \left\{\left|X_{j}^{\prime}\right|, d\right\}\right) \\
I^{\prime}{ }_{u s} & =\sum_{s^{\prime} \in \Phi_{U S} \backslash\{o\}} P_{U s}^{D L} h_{o, b}^{\prime} L_{0}\left(\max \left\{\left|X_{k}^{\prime}\right|, d\right\}\right)^{\prime}
\end{aligned}\right.
$$

where $I^{\prime}{ }_{m u}, I^{\prime}{ }_{u s}$ are the sum of the interference including the interference from the interfering tier 1 full-duplex users and interfering WTD tier 2 users, $g, h \sim \exp (1),|X|$ are the small-scale fading interfering channel power gain by mean 1 , the distance between interfering tier 1 users or tier 2 users $k^{\prime} \in \Phi_{U s} \backslash\{o\}$ (except the typical tier 2 user) and the typical tier 1 user based on the Rayleigh fading. 


\subsection{Transmit then Harvest}

On UL information transmission phase, in terms of the tier 1 users are the harvest then transmit users, the tier 2 WTDs will make use of the harvested energy as transmit power to transmit the data to tier 1 harvest then transmit relay on UL at the time slot $\frac{(1-\tau) T}{2}$, then the tier 1 harvest then transmit relay will transmit the information to the picocell BSs at the rest time slot $\frac{(1-\tau) T}{2}$, where $\tau \in(0,1)$. SINR received on the tier 1 harvest then transmit user from typical WTD tier 2 user can be given as

$$
\operatorname{SINR}_{U m}^{\prime}=\frac{P_{U S}^{\prime D L} h^{\prime}{ }_{o} L_{0}\left(\max \left\{\left|X_{o, u}^{\prime}\right|, d\right\}\right)}{I^{\prime}{ }_{u s}+\sigma^{2}}
$$

where $P_{U S}^{\prime D L}=\frac{E_{U S}}{(1-\tau) T}, h_{o}^{\prime} \sim \exp (1),\left|X_{o, u}^{\prime}\right|$ are the small-scale fading interfering channel power gain by mean 1, the distance between typical WTD tier 2 user and serving tier 1 user, respectively. In addition,

$$
I^{\prime}{ }_{u s}=\sum_{s^{\prime} \in \Phi_{U S} \backslash\{0\}} P_{U S}^{D L} h^{\prime}{ }_{o, b} L_{0}\left(\max \left\{\left|X_{k}^{\prime}\right|, d\right\}\right)
$$

the tier 1 relay users will only get the interference from the interfered tier 2 WTDs, where $I^{\prime}{ }_{u s}$ is the sum of the interference harvest from interfering WTD tier 2 users, $h \sim \exp (1),\left|X_{k}^{\prime}\right|$ are the small-scale fading interfering channel power gain by mean 1, the distance between interfering tier 2 users $k^{\prime} \in \Phi_{U S} \backslash\{0\}$ (except the typical tier 2 user), based on the Rayleigh fading. 


\section{Exact Analysis of DL and UL Transmission}

\subsection{Probability Density Function of Distance}

Suggesting $r$ is the distance between the tier 1 user and the picocell BS. Since the typical tier 1 user connect with the closest picocell BS, no other BS can be closer than R. The probability density function (pdf) of $x$ can be derived as

$$
\operatorname{Pr}(r>R)=e^{-\lambda \pi R^{2}}
$$

Therefore, the CDF is $\operatorname{Pr}(r \leq R)=F_{r}(R)=1-e^{-\lambda \pi R^{2}}$, the pdf can be denoted as $f_{r}(R)=\frac{d F_{r}(R)}{d r}=e^{-\lambda \pi r^{2}} 2 \pi \lambda r$

\subsection{Phase-1 DL Wireless Power Transfer}

\subsubsection{Full-duplex Relay}

On the DL full-duplex cooperative HetNet, 2-tier user harvest the directed energy and the RF at divided time slot $\tau \mathrm{T}$ as follow:

Theorem 1. The average harvested energy at 2-tier typical users in terms of tier 1 fullduplex relay are given by

$$
\begin{aligned}
\widetilde{\mathbb{E}}_{U m} & =\eta \tau T \beta\left(P_{B S}\left(1(x \leq d) d^{-\alpha_{B}}+1(x>d) x^{-\alpha_{B}}\right)\right. \\
& +2 \pi \lambda_{B S} P_{B S}\left(1(x \leq d)\left(\frac{1}{2} d^{-\alpha_{B}}\left(d^{2}-x^{2}\right)-\frac{d^{2-\alpha_{B}}}{2-\alpha_{B}}\right)\right. \\
& \left.\left.-1(x>d) \frac{x^{2-\alpha_{B}}}{2-\alpha_{B}}\right)+2 \pi \lambda_{B S} P_{M u}\left(\frac{1}{2} d^{2-\alpha_{U}}+\frac{d^{2-\alpha_{U}}}{\alpha_{U}-2}\right)\right)
\end{aligned}
$$


and

$$
\begin{aligned}
\widetilde{\mathbb{E}}_{U s} & =\eta \tau T \beta\left(P_{U m}\left(1(y \leq d) d^{-\alpha_{U}}+1(y>d) y^{-\alpha_{U}}\right)\right. \\
& +2 \pi \lambda_{B S} P_{U m}\left(1(y \leq d)\left(\frac{1}{2} d^{-\alpha_{U}}\left(d^{2}-y^{2}\right)-\frac{d^{2-\alpha_{U}}}{2-\alpha_{U}}\right)\right. \\
& \left.\left.-1(y>d) \frac{y^{2-\alpha_{U}}}{2-\alpha_{U}}\right)+2 \pi \lambda_{B S} P_{B S}\left(\frac{1}{2} d^{2-\alpha_{B}}+\frac{d^{2-\alpha_{B}}}{\alpha_{B}-2}\right)\right)
\end{aligned}
$$

proof: See A Proof of Theorem 1.

where $\widetilde{\mathbb{E}}_{U m}, \widetilde{\mathbb{E}}_{U s}$ are the total energy can be harvested on the DL on tier 1 full-duplex relay users, and tier 2 WTDs users given by (1), (2), (3), follows the Campbell's theorem $\boldsymbol{E}\left\{\sum_{x_{i} \in \Phi} f\left(x_{i}\right)\right\}=\lambda \int \boldsymbol{E}\{f(x)\} d x$.

The average harvested energy for a typical tier 1 full-duplex user that is associated with a closest picocell BS. From (11), the average harvested energy can be denoted as $\bar{E}(x)=\int_{0}^{\infty} \tilde{E}(x) f(x) d x$. We set the distance between 2-tier users as a constant value. Imagining that tier 2 WTDs just in the pockets or bags, connect to the smart phone in your hand, so the distance is close enough to be assumed as a constant value $d_{0}$.

\subsubsection{Harvest then Transmit}

In terms of the tier 1 users are the harvest then transmit relay users, the total energy that the tier 2 WTDs users harvest on the DL at the time slot of $\tau T$, can be given as

$$
\begin{aligned}
\widetilde{\mathbb{E}}_{U s}^{\prime} & =\eta \tau T \beta\left(P_{U m}\left(1(y \leq d) d^{-\alpha_{U}}+1(y>d) y^{-\alpha_{U}}\right)\right. \\
& \left.+2 \pi \lambda_{B S} P_{B S}\left(\frac{1}{2} d^{2-\alpha_{B}}+\frac{d^{2-\alpha_{B}}}{\alpha_{B}-2}\right)\right)
\end{aligned}
$$

proof: See A Proof of Theorem 1.

where $\widetilde{\mathbb{E}}_{\boldsymbol{U} \text { s }}^{\prime}$ is the total energy can be harvested on the DL receive on tier 2 WTDs users 
given by (4), (5), follows the Campbell's theorem $\boldsymbol{E}\left\{\sum_{x_{i} \in \Phi} f\left(x_{i}\right)\right\}=\lambda \int \boldsymbol{E}\{f(x)\} d x$. We set the distance between 2-tier users as a constant value. Imagining that tier 2 WTDs just in the pockets or bags, connect to the smart phone in your hand, so the distance is close enough to be assumed as a constant value $d_{0}$.

\subsection{Phase-2 UL Information Transmission}

\subsubsection{Full-duplex Relay}

On the UL full-duplex cooperative HetNet, the WTDs tier 2 users transmit the information signal with a special transmit power that harvested from the DL at the divided same time slot $(1-\tau) T[15]$.

Theorem 2. The average ergodic rate received at tier 1 full-duplex user is given by

$$
\begin{gathered}
R_{U m}^{\text {Exact }}=\mathbb{E}_{\operatorname{SINR}_{U m}}\left\{(1-\tau) T \operatorname{Tl}\left(1+\operatorname{SINR}_{U m}\right)\right\} \\
\quad \text { (a) } \int_{R_{1}>0}^{\infty} \operatorname{Pr}\left(\operatorname{SINR}_{U m}>t_{1}\right) d R_{1}
\end{gathered}
$$

where $t_{1}=e^{R_{1} /(1-\tau) T}-1, \mathrm{R}_{1}$ is the throughput threshold, then from (6), (7) we can derive the coverage probability of $\operatorname{SINR}_{U m}$, where equals to the complementary cumulative distribution function (CCDF) of SINR as

$$
\begin{aligned}
\operatorname{Pr}\left(\operatorname{SINR}_{U m}>t_{1}\right) & =\operatorname{Pr}\left(\frac{P_{U s}^{D L} h_{o}^{\prime} L_{0}\left(\max \left\{\left|X_{o, u}^{\prime}\right|, d\right\}\right)}{I^{\prime}{ }_{m u}+I^{\prime}{ }_{u s}+\sigma^{2}}>t_{1}\right) \\
& =\operatorname{Pr}\left(h^{\prime}{ }_{o}>t_{1} \Delta_{U s}{ }^{-1}\left(I^{\prime}{ }_{m u}+I^{\prime}{ }_{u s}+\sigma^{2}\right) d^{\alpha_{U}}\right) \\
& =\mathcal{L}_{I^{\prime}{ }_{m u}}\left(t_{1} \Delta_{U s}{ }^{-1} d^{\alpha_{U}}\right) \cdot \mathcal{L}_{I^{\prime}{ }_{u s}}\left(t_{1} \Delta_{U s}{ }^{-1} d^{\alpha_{U}}\right) \\
& \cdot \exp \left(-t_{1} \sigma^{2} \Delta_{U s}{ }^{-1} d^{\alpha_{U}}\right)
\end{aligned}
$$




$$
\begin{aligned}
& =\exp \left(-2 \pi \lambda_{B s}\left(\frac{t_{1} \Delta_{U s}{ }^{-1} \Delta_{U m}}{1+t_{1} \Delta_{U s}{ }^{-1} \Delta_{U m}} \frac{1}{2} d^{2}\right.\right. \\
& \left.\left.+\frac{t_{1} \Delta_{U s}{ }^{-1} \Delta_{U m} d^{2}}{\alpha_{U}-2} 2 F 1\left[1, \frac{\alpha_{U}-2}{\alpha_{U}}, 2-\frac{2}{\alpha_{U}},-t_{1} \Delta_{U s}{ }^{-1} \Delta_{U m}\right]\right)\right) \\
& \cdot \exp \left(-2 \pi \lambda_{B s}\left(\frac{t_{1}}{1+t_{1}} \frac{1}{2}\left(\mathrm{~d}^{2}-d_{0}^{2}\right)\right.\right. \\
& \left.\left.+\frac{t_{1} d^{2}}{\alpha_{U}-2} 2 F 1\left[1, \frac{\alpha_{U}-2}{\alpha_{U}}, 2-\frac{2}{\alpha_{U}},-t_{1}\right]\right)\right) \\
& \cdot \exp \left(-t_{1} \sigma^{2} \Delta_{U s}{ }^{-1} d^{\alpha_{U}}\right)
\end{aligned}
$$

where $\Delta_{U s}=P_{U S}^{D L} \beta=\frac{E_{U S}}{(1-\tau) T} \beta, \Delta_{U m}=P_{U m}^{D L} \beta=\frac{E_{U m}}{(1-\tau) T} \beta$, the tier 2 WTDs is close enough with tier 1 user, so there is no user association for typical tier 2 user, we set as reference distance $d=1$. Therefore, the UL outage probability, where shows the user who is not in the coverage and its SINR is below than the threshold [16] is given by

$$
\begin{aligned}
P_{\text {out }_{U m}^{U L}} & =1-\operatorname{Pr}\left(\operatorname{SINR}_{U m}(x)>t_{1}\right) \\
& =1-\exp \left(-2 \pi \lambda_{B s}\left(\frac{t_{1} \Delta_{U s}{ }^{-1} \Delta_{U m}}{1+t_{1} \Delta_{U s}{ }^{-1} \Delta_{U m}} \frac{1}{2} d^{2}\right.\right. \\
& \left.\left.+\frac{t_{1} \Delta_{U s}{ }^{-1} \Delta_{U m} d^{2}}{\alpha_{U}-2} 2 F 1\left[1, \frac{\alpha_{U}-2}{\alpha_{U}}, 2-\frac{2}{\alpha_{U}},-t_{1} \Delta_{U s}{ }^{-1} \Delta_{U m}\right]\right)\right) \\
& \cdot \exp \left(-2 \pi \lambda_{B S}\left(\frac{t_{1}}{1+t_{1}} \frac{1}{2}\left(\mathrm{~d}^{2}-d_{0}^{2}\right)\right.\right. \\
& \left.\left.+\frac{t_{1} d^{2}}{\alpha_{U}-2} 2 F 1\left[1, \frac{\alpha_{U}-2}{\alpha_{U}}, 2-\frac{2}{\alpha_{U}},-t_{1}\right]\right)\right) \\
& \cdot \exp \left(-t_{1} \sigma^{2} \Delta_{U s}{ }^{-1} d^{\alpha_{U}}\right)
\end{aligned}
$$

In addition,

$$
\mathcal{L}_{I^{\prime}{ }^{\prime} \text { uu }}\left(t_{1} \Delta_{U s}{ }^{-1} d^{\alpha_{U}}\right)
$$




$$
\begin{aligned}
& =\exp \left(-2 \pi \lambda_{B s}\left(\frac{t_{1} \Delta_{U s}{ }^{-1} \Delta_{U m}}{1+t_{1} \Delta_{U s}{ }^{-1} \Delta_{U m}} \frac{1}{2} d^{2}\right.\right. \\
& \left.\left.+\frac{t_{1} \Delta_{U s}{ }^{-1} \Delta_{U m} d^{2}}{\alpha_{U}-2} 2 F 1\left[1, \frac{\alpha_{U}-2}{\alpha_{U}}, 2-\frac{2}{\alpha_{U}},-t_{1} \Delta_{U s}{ }^{-1} \Delta_{U m}\right]\right)\right)
\end{aligned}
$$

where

$$
\begin{aligned}
\mathcal{L}_{I^{\prime} m u}(s) & =\mathbb{E}_{I^{\prime} m u}\left\{\exp \left(-s \sum P_{U m}^{D L} g_{m, o}^{\prime} L_{0}\left(\max \left\{\left|X_{j}^{\prime}\right|, d\right\}\right)\right)\right\} \\
& (b) \exp \left(-\lambda_{B s} \int_{R^{2}}\left(1-\mathcal{L}_{g_{m, o}^{\prime}}\left(s P_{U m}^{D L} L_{0}\left(\max \left\{\left|X_{j}^{\prime}\right|, d\right\}\right)\right)\right) d x\right) \\
= & (c) \exp \left(-2 \pi \lambda_{B s}\left(\frac{s \Delta_{U m} d^{-\alpha_{U}}}{1+s \Delta_{U m} d^{-\alpha_{U}}} \frac{1}{2} d^{2}\right.\right. \\
= & \\
+ & \left.\left.\frac{s \Delta_{U m} d^{\left(2-\alpha_{U}\right)}}{\alpha_{U}-2} 2 F 1\left[1, \frac{\alpha_{U}-2}{\alpha_{U}}, 2-\frac{2}{\alpha_{U}},-s \Delta_{U m} d^{-\alpha_{U}}\right]\right)\right)
\end{aligned}
$$

and

$$
\begin{array}{r}
\mathcal{L}_{I^{\prime}{ }_{\text {us }}}\left(t_{1} \Delta_{U s}{ }^{-1} d^{\alpha_{U}}\right)=\exp \left(-2 \pi \lambda_{B s}\left(\frac{t_{1}}{1+t_{1}} \frac{1}{2}\left(\mathrm{~d}^{2}-d_{0}^{2}\right)\right.\right. \\
\left.\left.+\frac{t_{1} d^{2}}{\alpha_{U}-2} 2 F 1\left[1, \frac{\alpha_{U}-2}{\alpha_{U}}, 2-\frac{2}{\alpha_{U}},-t_{1}\right]\right)\right)
\end{array}
$$

where

$$
\begin{aligned}
\mathcal{L}_{I^{\prime}{ }_{u s}}(s) & =\mathbb{E}_{I^{\prime}{ }_{m u}}\left\{\exp \left(-s \sum_{s^{\prime} \in \phi_{U s} \backslash\{o\}} P_{U s}^{D L} h_{o, b}^{\prime^{\prime}} L_{0}\left(\max \left\{\left|X_{k}^{\prime}\right|, d\right\}\right)\right)\right\} \\
& (d) \exp \left(-\lambda_{B s} \int_{R^{2}}\left(1-\mathcal{L}_{h_{o, b}^{\prime}}\left(s P_{U s}^{D L} L_{0}\left(\max \left\{\left|X_{k}^{\prime}\right|, d\right\}\right)\right)\right) d x\right) \\
& =\operatorname{le} \exp \left(-2 \pi \lambda_{B s}\left(\frac{s \Delta_{U s} d^{-\alpha_{U}}}{1+s \Delta_{U s} d^{-\alpha_{U}}} \frac{1}{2}\left(d^{2}-d_{0}^{2}\right)\right.\right.
\end{aligned}
$$




$$
\left.\left.+\frac{s \Delta_{U S} d^{\left(2-\alpha_{U}\right)}}{\alpha_{U}-2} 2 F 1\left[1, \frac{\alpha_{U}-2}{\alpha_{U}}, 2-\frac{2}{\alpha_{U}},-s \Delta_{U S} d^{-\alpha_{U}}\right]\right)\right)
$$

proof: See A Proof of Theorem 2.

based on $\mathbb{E}\left\{\prod_{x \in \Phi} f(x)\right\}=\exp \left(-\lambda \int_{R^{2}}(1-f(x)) d x\right)$, and $\mathcal{L}_{I^{\prime}{ }_{m u}}(),. \mathcal{L}_{I^{\prime} \text { us }}($.$) are$ the Laplace transform of the PDF of $I^{\prime}{ }_{m u}, I^{\prime}{ }_{u s}$, and can be derived by making use of the generating function of PPP [17].

\subsubsection{Harvest then Transmit}

In terms of the tier 1 users are the harvest then transmit relay users, the WTDs tier 2 users transmit the information signal with a special transmit power that harvested from the DL at the divided time slot $\frac{(1-\tau) T}{2}$.

$$
\begin{aligned}
R_{U m}^{\prime \mathrm{Exact}} & =\mathbb{E}_{\mathrm{SINR}_{U m}^{\prime}}\left\{\frac{(1-\tau) T}{2} \ln \left(1+\mathrm{SINR}_{U m}^{\prime}\right)\right\} \\
& (f) \int_{R_{1}>0}^{\infty} \operatorname{Pr}\left(\mathrm{SINR}_{U m}^{\prime}>t_{2}\right) d R_{1} \\
& ={ }^{\infty}
\end{aligned}
$$

where $t_{2}=e^{R_{1} / \frac{(1-\tau) T}{2}}-1, \mathrm{R}_{1}$ is the throughput threshold, then from (8), (9) we can derive the coverage probability of $\operatorname{SINR}_{U m}^{\prime}$ as

$$
\begin{aligned}
\operatorname{Pr}\left(\operatorname{SINR}_{U m}^{\prime}>t_{2}\right) & =\operatorname{Pr}\left(\frac{P_{U s}^{\prime D L} h^{\prime}{ }_{o} L_{0}\left(\max \left\{\left|X_{o, u}^{\prime}\right|, d\right\}\right)}{I^{\prime}{ }_{u s}+\sigma^{2}}>t_{1}\right) \\
& =\operatorname{Pr}\left(h^{\prime}{ }_{o}>t_{1} \Delta_{U s}{ }^{-1}\left(I^{\prime}{ }_{u s}+\sigma^{2}\right) d^{\alpha_{U}}\right) \\
& =\mathcal{L}_{I^{\prime}{ }_{u s}}\left(t_{1} \Delta_{U s}{ }^{-1} d^{\alpha_{U}}\right) \cdot \exp \left(-t_{1} \sigma^{2} \Delta_{U s}{ }^{-1} d^{\alpha_{U}}\right) \\
& =\exp \left(-2 \pi \lambda_{B s}\left(\frac{t_{1}}{1+t_{1}} \frac{1}{2}\left(\mathrm{~d}^{2}-d_{0}^{2}\right)\right.\right.
\end{aligned}
$$




$$
\begin{aligned}
& \left.\left.+\frac{t_{1} d^{2}}{\alpha_{U}-2} 2 F 1\left[1, \frac{\alpha_{U}-2}{\alpha_{U}}, 2-\frac{2}{\alpha_{U}},-t_{1}\right]\right)\right) \\
& \cdot \exp \left(-t_{1} \sigma^{2} \Delta_{U s}{ }^{-1} d^{\alpha_{U}}\right)
\end{aligned}
$$

where, $\Delta_{U S}^{\prime}=P_{U S}^{\prime D L} \beta=\frac{E_{U s}^{\prime}}{\frac{(1-\tau) r}{2}} \beta$, the tier 2 WTDs is close enough with tier 1 user, so there is no user association for typical tier 2 user, we set as reference distance $d=1$. Therefore, the UL outage probability [15] is given by

$$
\begin{aligned}
P_{\text {out }_{U m}}^{\text {UL }} & =1-\operatorname{Pr}\left(\operatorname{SINR}_{U m}^{\prime}(x)>t_{2}\right) \\
& =1-\exp \left(-2 \pi \lambda_{B S}\left(\frac{t_{1}}{1+t_{1}} \frac{1}{2}\left(\mathrm{~d}^{2}-d_{0}^{2}\right)\right.\right. \\
& \left.\left.+\frac{t_{1} d^{2}}{\alpha_{U}-2} 2 F 1\left[1, \frac{\alpha_{U}-2}{\alpha_{U}}, 2-\frac{2}{\alpha_{U}},-t_{1}\right]\right)\right) \\
& \cdot \exp \left(-t_{1} \sigma^{2} \Delta_{U s}{ }^{-1} d^{\alpha_{U}}\right)
\end{aligned}
$$

Proof: See A Proof of Theorem 2.

\subsection{Numerical Results}

In this section, we use the analytical expressions of the average received energy, the UL average ergodic rate, and UL outage probability to evaluate the full-duplex relay system network in the two situations of tier 1 users are the full-duplex relay and harvest then transmit relays. The exact results are validated by the method of Monte Carlo, where random the distance of each tier users with the density $\lambda_{B S}=10^{-3} \mathrm{~m}^{-2}, \alpha_{B}=$ 3.5, $\alpha_{U}=3, d=1, R_{1}=0.1$. For all the numerical results, $P_{B S}=33 \mathrm{dBm}, P_{B S}=$ $25 \mathrm{dBm}$. 


\subsubsection{Full-duplex Relay}

Fig. 8, 9, plot the effect of allocated time slot factor for energy harvesting phrase to the average harvested energy. In terms of the tier 1 users are the full-duplex relay users, it is observed that the harvested energy divided into the direct WPT and from the ambient RF on the time slot $\tau T$. The average harvested energy increase with the increasing of the allocated time slot for energy harvesting. The energy harvest directly from the signal is larger than the energy harvest from the ambient RF.

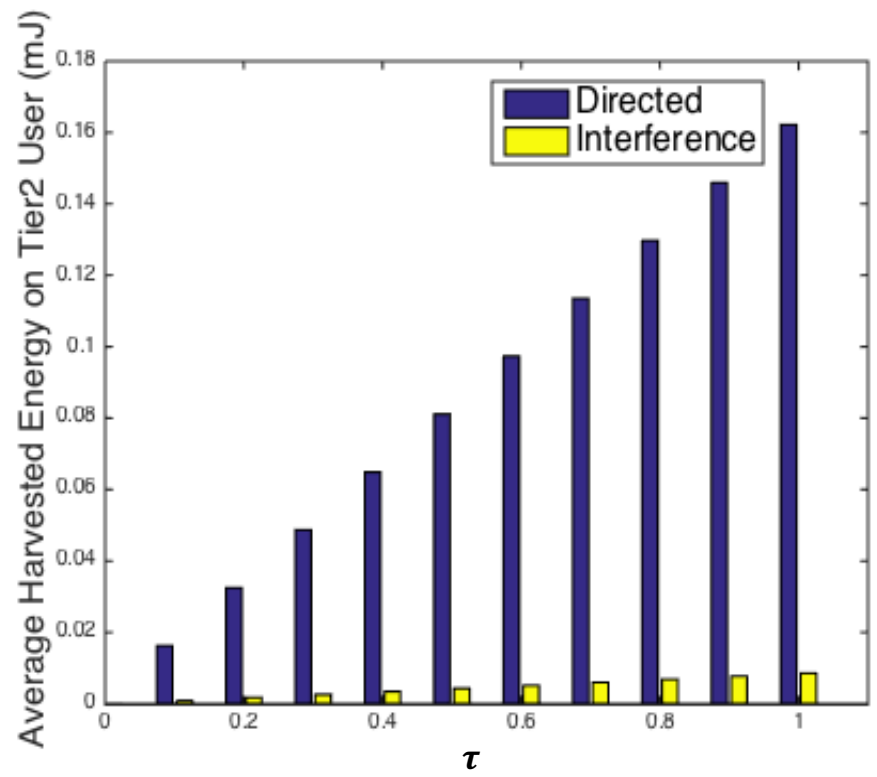

Fig. 8. The Average Harvested Energy on Tier 2 User with full-duplex relay 


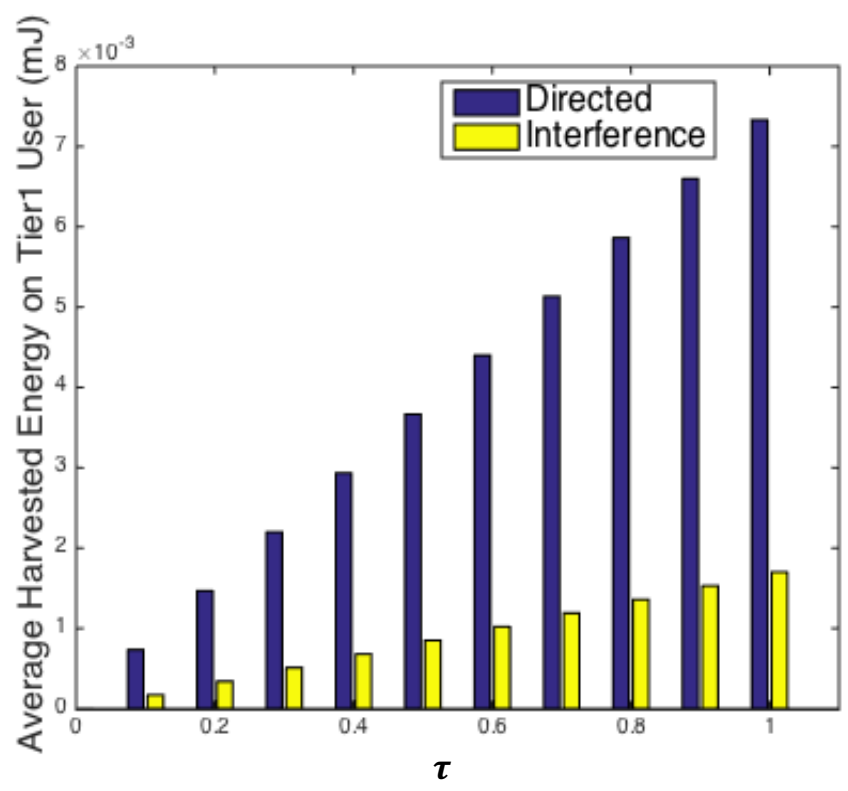

Fig. 9. The Average Harvested Energy on Tier 1 User with full-duplex relay

Fig.10 plots the effect of transmit power and the density $\lambda_{\mathrm{Bs}}$ to the average harvested energy of 2-tier users. With the increasing of the density of picocell BS and transmit power, the harvested energy receive on both 2-tier users will increase. That is because the directed energy and the interference from the picocell BSs increase, the more energy can be harvested on DL. When the transmit power of picocell BS are little bigger than the tier 1 user, the average harvested energy are not significantly improved. 


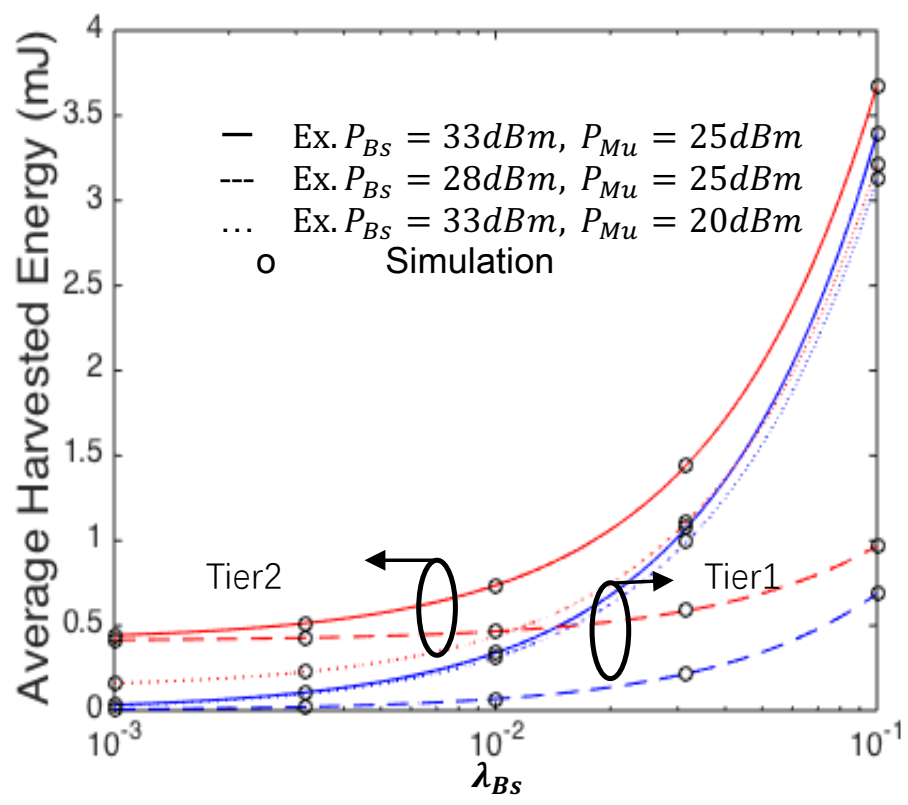

Fig. 10. The Average Harvested Energy on 2-Tier User with full-duplex relay

Fig. 11 depicts the impact of density of the picocell BSs and the RF to DC efficiency to the average harvested energy. It is observed that with the increasing of the RF to DC efficiency, tier 2 WTDs will harvest more energy because more radio frequency will transform to the direct current energy.

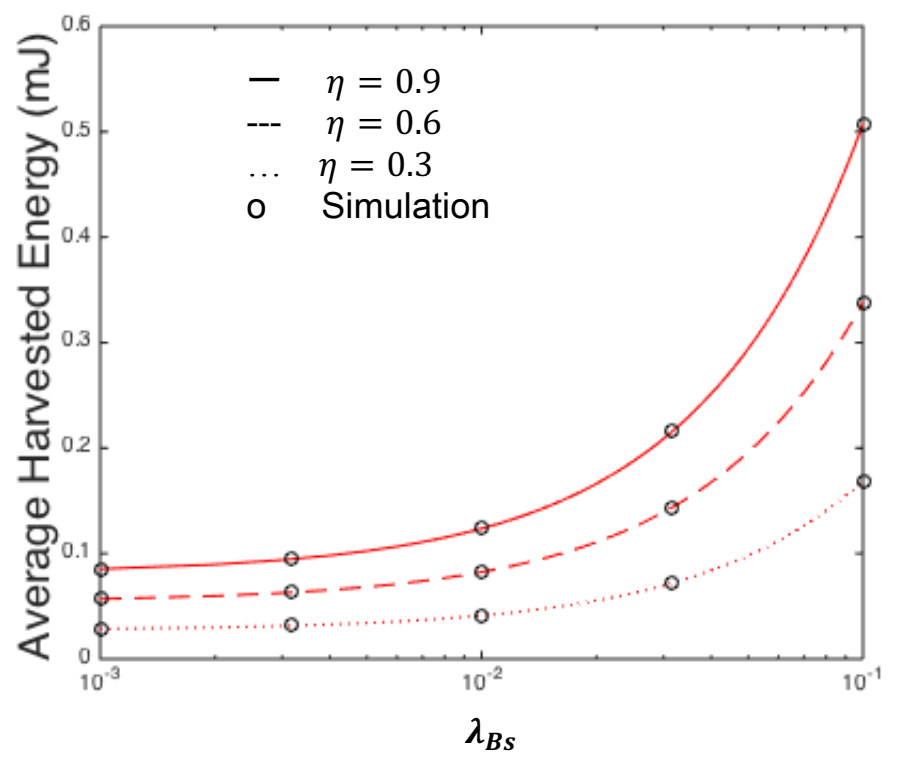

Fig. 11. The Average Harvested Energy on Tier 2 User with full-duplex relay

Fig. 12 plots the effect of density of picocell BS and allocated time slot factor for 
energy harvesting to the average ergodic rate receive on tier 1users. With the increasing of the $\lambda_{\mathrm{Bs}}$, the average ergodic rate decrease, due to the tradeoff between the increasing $\lambda_{\mathrm{Bs}}$ and the transmit power. It is observed that the density of picocell BSs increase, the interference from the picocell BSs on UL will be worse. Because of the less allocated time slot for DL energy harvesting, the less transmit power for UL information transmission, the average ergodic rate will decrease significantly.

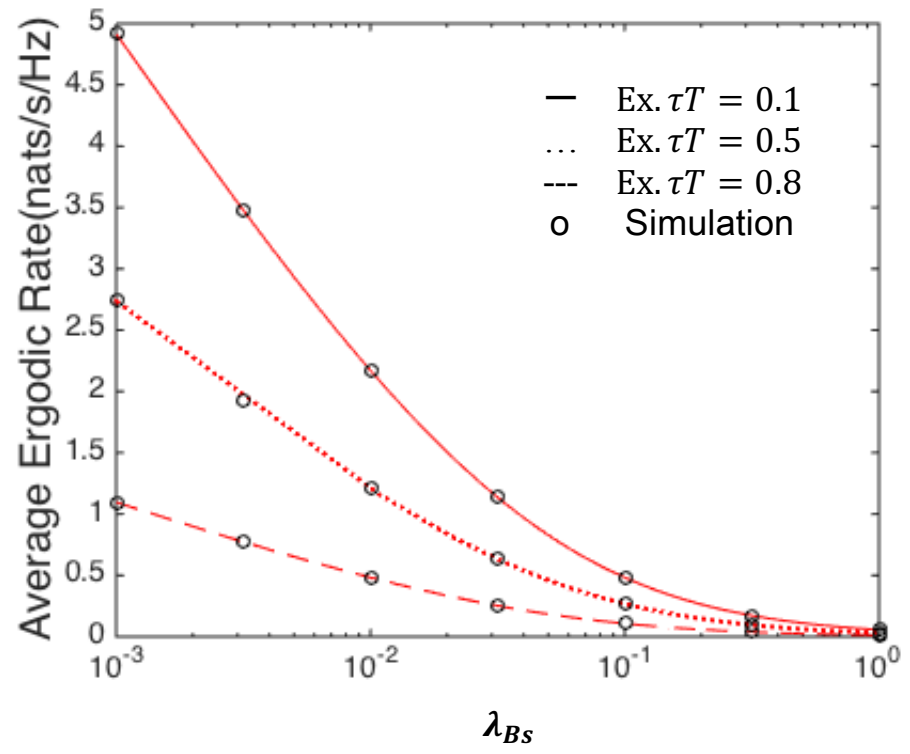

Fig. 12. The Average Ergodic Rate of tier 2 WTD on UL with full-duplex relay

Fig.13 plots the effect of allocated time slot for energy harvesting and path loss exponent to the outage probability. The UL outage probability will improve then degrade due to the tradeoff between the benefits from the increasing allocated time slot for energy harvesting and the less time left for information transmission. The UL outage probability degrades significantly due to the more allocated time slot for DL energy harvesting, the transmit power will improve, but there is less time for UL information transmission, moreover, interference from the other users will be worse due to the 
increasing transmit power. It is observed that the smaller path loss exponent, the better UL performance, the UL outage probability will improve.

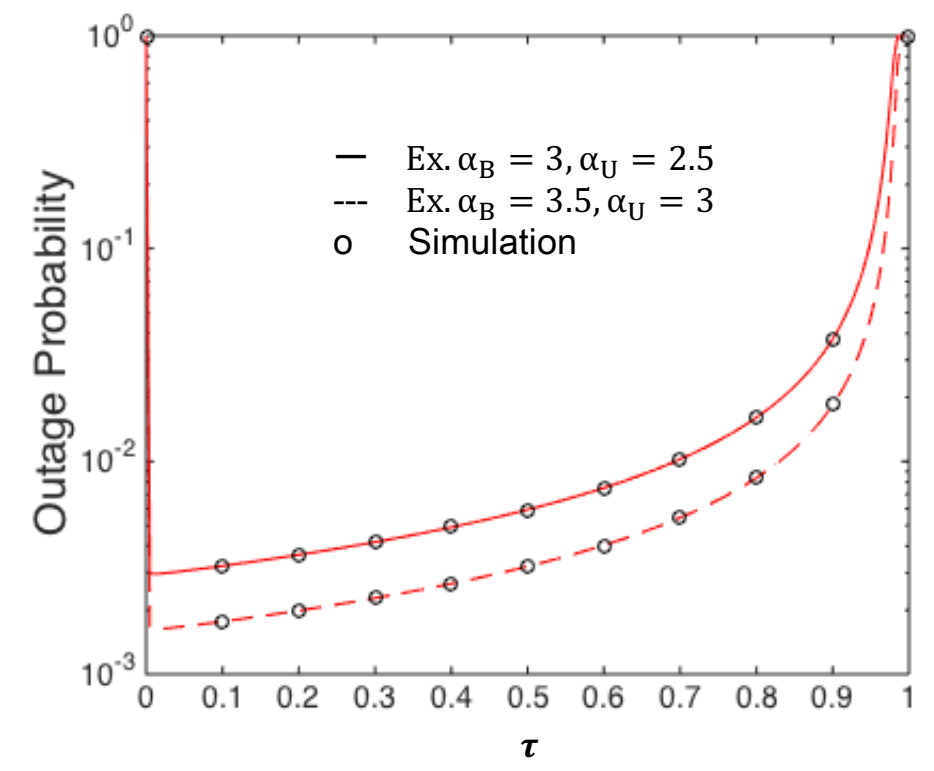

Fig. 13. The UL outage probability of tier 2 WTD with full-duplex relay

Fig.14 plots the effect of the density of the picocell BSs and the path loss exponent to the UL outage probability. It is observed that with the increasing of the density of the picocell BSs, the UL outage probability of the WTDs will degrade significantly. The path loss exponent becomes smaller, the outage probability will become worse owe to the interference from the interfered users will increase, the performance of UL will be worser. 


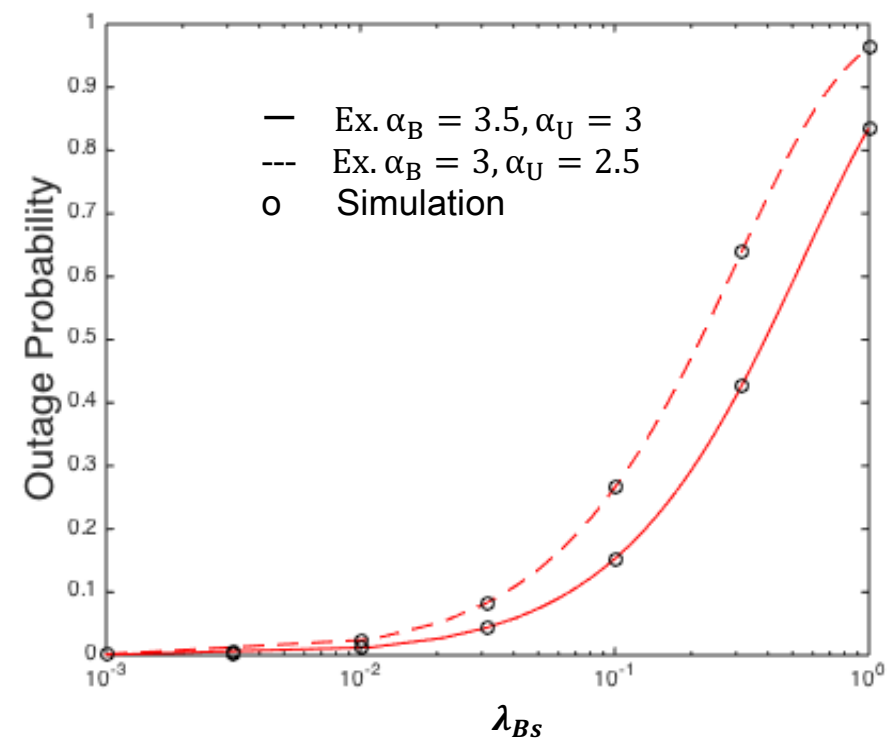

Fig. 14. The UL outage probability of tier 2 WTD with full-duplex relay

\subsubsection{Harvest then Transmit}

Fig. 15 plots the effect of time slot factor $\tau T$ to the average harvested energy on tier 2 WTDs users, where $\tau T=0.5$. It is observed that the harvested energy can be divided into the energy harvest from the DL directly and the energy from the ambient RF. The energy harvest directly from the tier 1 relay users will much more than the energy harvest from the interference.

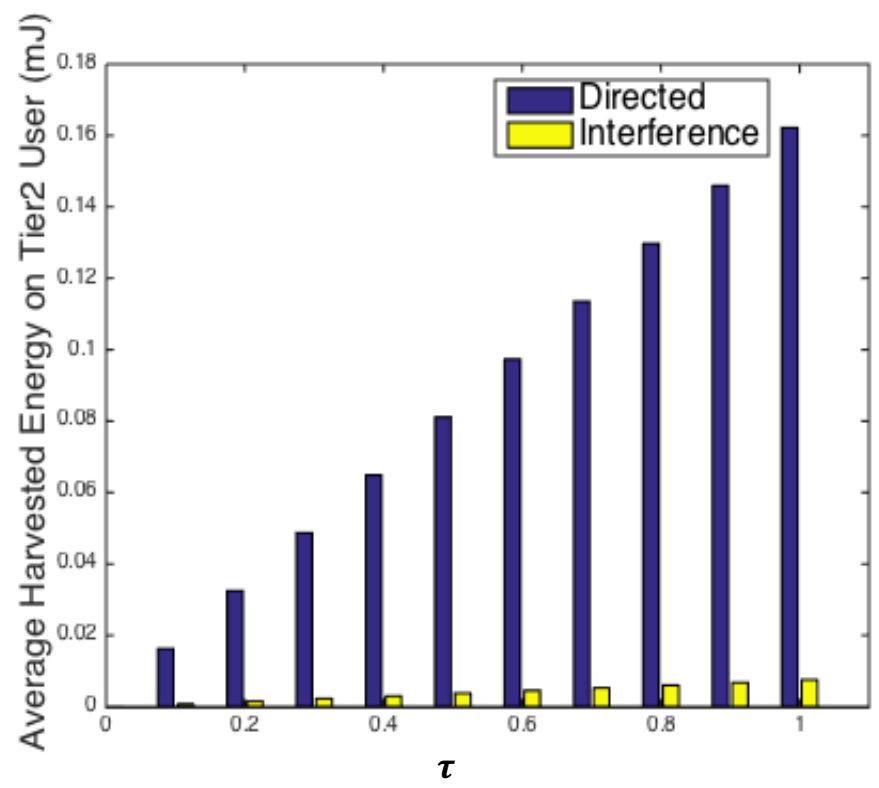

Fig. 15. The Average Harvested Energy on Tier 2 User with harvest then transmit relay

Fig.16 plots the comparison of average harvested energy between the situation of 
full-duplex relay and harvest then transmit relay on the effect of density of picocell BSs and the transmit power on DL. It is observed that in the situation of tier 1 users are harvest then transmit relay, the harvested energy is less than the situation of tier 1 are full-duplex relay. Moreover, with the decreasing of the transmit power on DL and the density of picocell BSs, the less energy can be harvest.

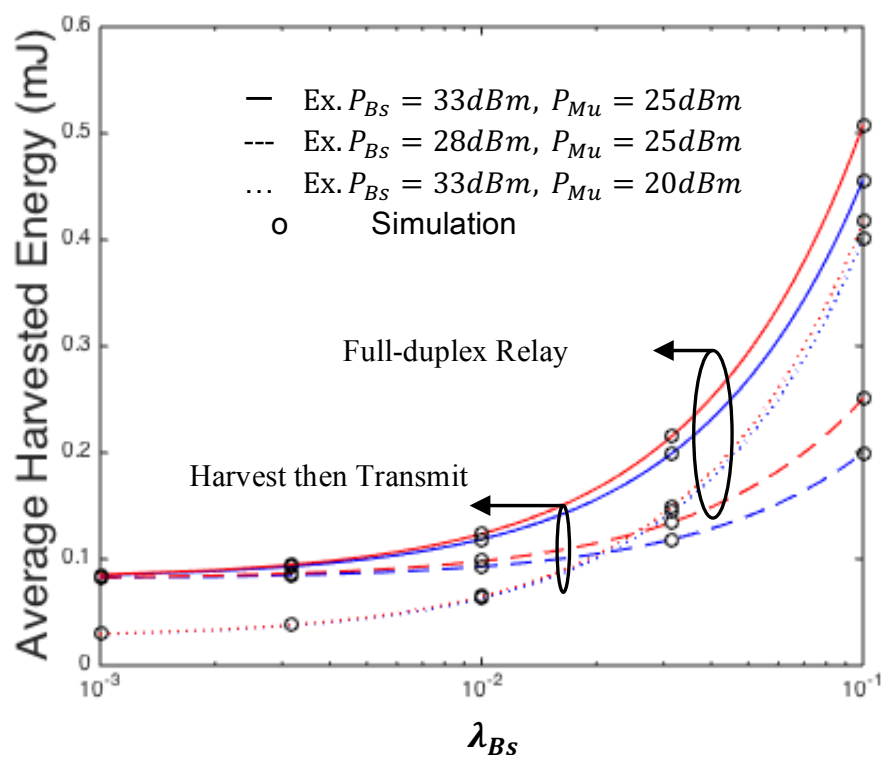

Fig. 16. The comparison of Average Harvested Energy between the the full-duplex relay and harvest then transmit relay with transmit power

Fig. 17 depicts the comparison of average harvested energy between the situation of full-duplex relay and harvest then transmit relay on the effect of the density of picocell BSs and the RF to DC efficiency to the average harvested energy. It is observed that in the situation of tier 1 users are the full-duplex relay, the more energy can be harvested than the situation of tier 1 users are the harvest then transmit relay owe to the interference from the ambient RF increase. With the increasing of RF to DC transform efficiency, more energy can be harvested. When the density of picocell BSs increase to $\lambda_{B S}=10^{-2} \mathrm{~m}^{-2}$, the average harvested energy is almost the same. 


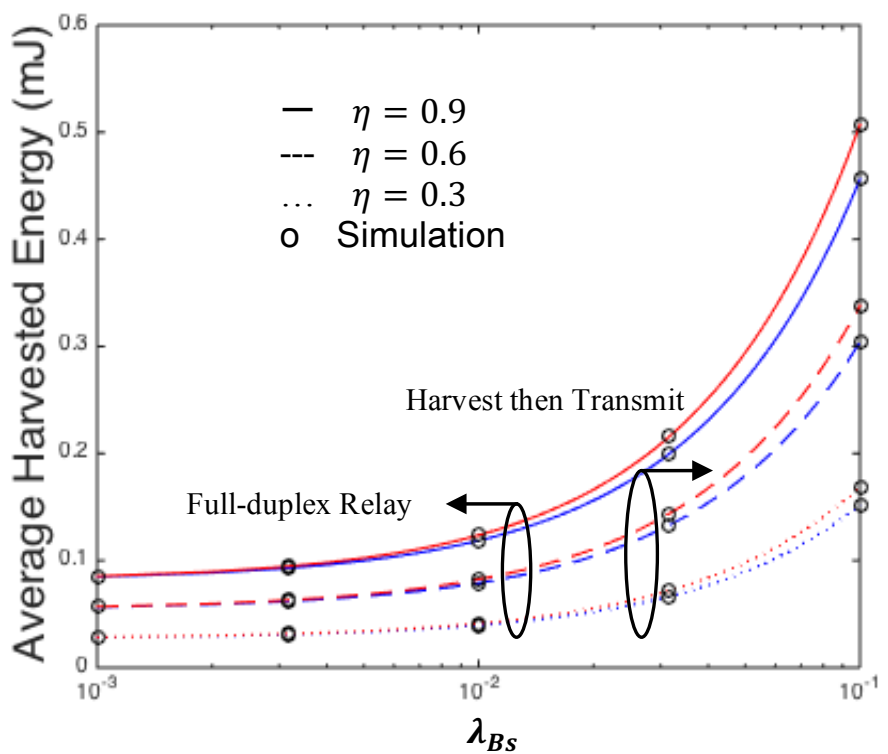

Fig. 17. The comparison of Average Harvested Energy between the full-duplex relay and harvest then transmit relay with RF to DC transform efficiency

Fig.18 plots the comparison of average ergodic rate between the situation of fullduplex relay and the harvest then transmit relay on the impact of the density of picocell BSs and the time slot factor $\tau T=0.5$ for energy harvesting process. In the situation of tier 1 users are the full-duplex relay, the rest of time slot for information transmission process is $(1-\tau) T$, moreover, in the situation of tier 1 users are the harvest then transmit relay, the rest of the time slot for information transmission process is $\frac{(1-\tau) T}{2}$. It is observed that with the increasing of the time slot for energy harvesting process, the average ergodic rate degrades significantly. Compare to the situation of tier 1 users are the full-duplex relay the average ergodic rate is worser than in the situation of tier 1 users are the harvest then transmit relay owe to the tradeoff benefits between the harvested energy and the interference, the harvested energy is less, the transmit power is less, moreover the time slot left for information is less, the average ergodic rate is worse. 


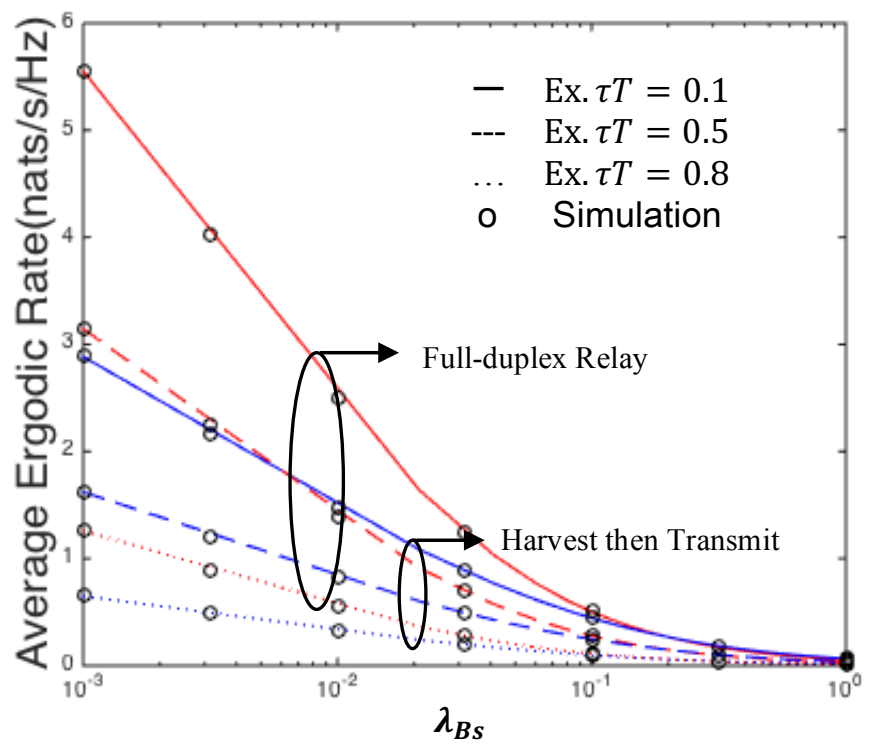

Fig. 18. The comparison of Average Ergodic Rate between the full-duplex relay and harvest then transmit relay with time slot

Fig. 19 plots the comparison of outage probability between the situation of fullduplex relay and the harvest then transmit relay on the effect of the time slot factor $\tau T$, where $\tau T=0.5$, and the path loss exponent. It is observed that compare to the situation of tier 1 users are the harvest then transmit relay, the UL outage probability is worser than in the situation of tier 1 users are the full-duplex owe to the tradeoff benefits between the transmit power on UL and the time slot left for information transmission process. With the path loss exponent are smaller, the better UL performance, but the interference increases either, the outage probability degrades. 


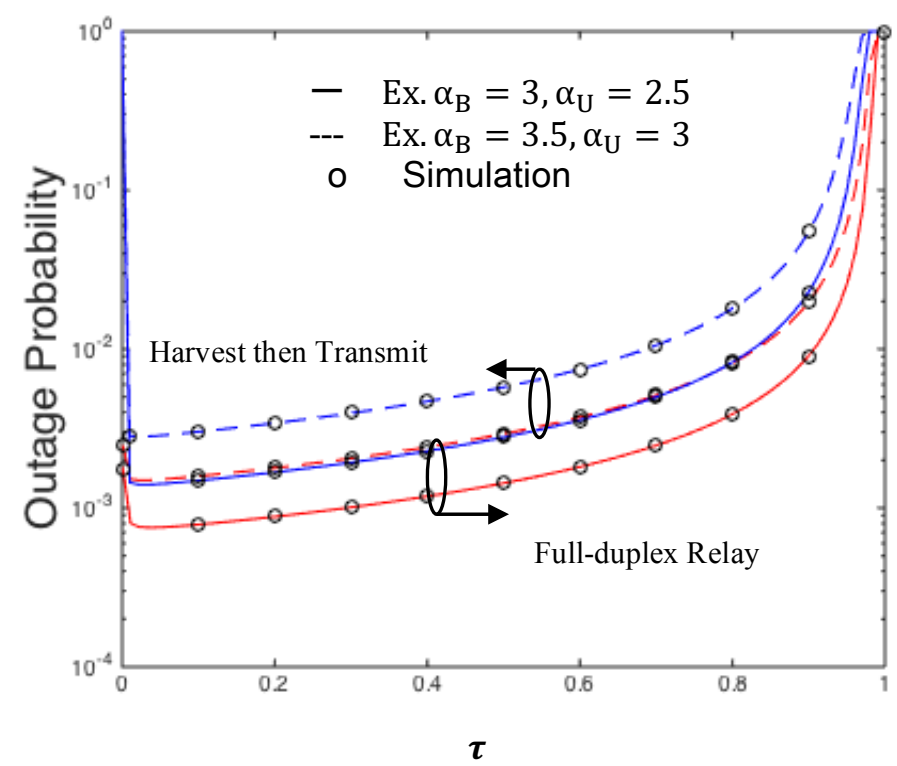

Fig. 19. The comparison of Outage Probability between the full-duplex relay and harvest then transmit relay with path loss exponent

Fig.20 plots the comparison of outage probability between the situation of fullduplex relay and the harvest then transmit relay on the effect of density of picocell BSs and the path loss exponent. It is observed that the situation of tier 1 users are the harvest then transmit relay, the UL outage probability will worse than the situation of tier 1 users are the full-duplex relay then better with the increasing of the density of picocell BSs owe to the tradeoff benefits between the transmit power and the interference from the interfered users. With the path loss exponent are smaller, the better UL performance, but the interference increases either, the outage probability degrades. 


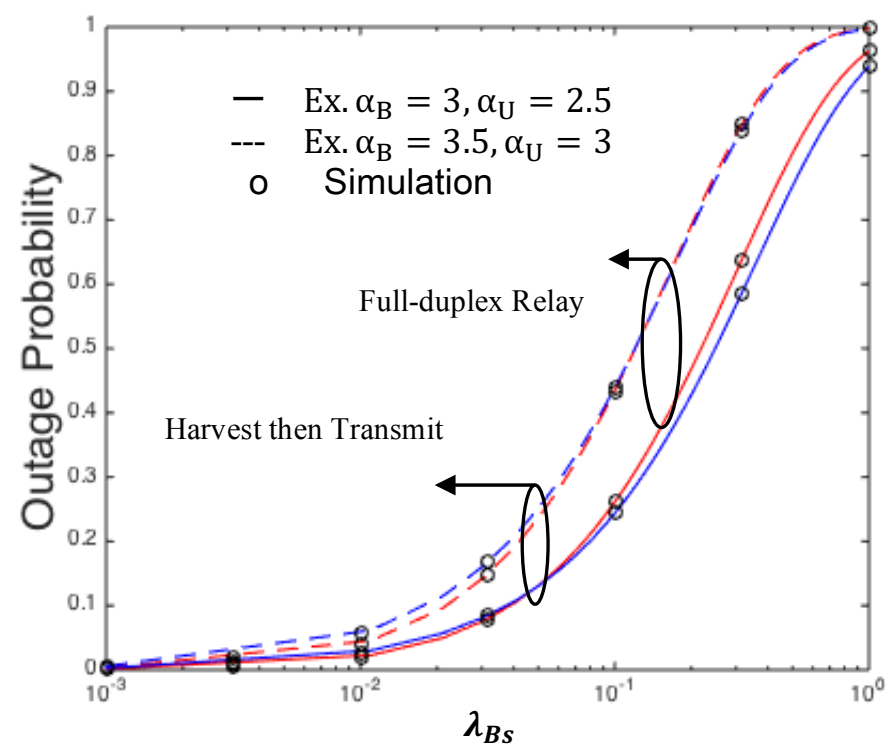

Fig. 20. The comparison of Outage Probability between the full-duplex relay and harvest then transmit relay with path loss exponent 


\section{CONCLUSIONS}

In this research, we examine a tractable model for multi-hop HetNet system with two kinds of tier 1 relay user, tier 1 users are full-duplex cooperative relay and tier 1 users are harvest then transmit relay. In the situation of the tier 1 user are the full-duplex relay, tier 1 relay users and the WTDs tier 2 users can be charged on DL at the same allocated time slot $\tau T$, then tier 1 user will support tier 2 user transmit the information on UL at the same allocated time slot $(1-\tau) T$. In the situation of tier 1 users are harvest then transmit relay, the WTDs tier 2 users can be charged on DL at the same allocated time slot $\tau T$, then tier 1 user will support tier 2 user transmit the information on UL at the same allocated time slot $\frac{(1-\tau) T}{2}$. By the distribution of HPPP with particular density, we derive the equations of the average harvested energy, the average ergodic rate and UL outage probability. It is observed that increasing with the allocated time slot for energy harvesting and the density of picocell BSs, the average harvested energy will increase. Due to the tradeoff between the increasing of the allocated time slot for transmit power and impact from increasing density of the picocell BSs, the less time left for information transmission, the average ergodic rate of WTDs and the UL outage probability will degrade. Compare two kinds of relay method, the performance of the situation of tier 1 users are full-duplex relay is better than the situation of tier 1 users are harvest then transmit relay. 


\section{Future Work}

Along with the deployment of HetNet, the complicated distribution of acess point and users will be the problem for analyzing the performance of the network. UDN is a new definition to indicates that there are more cells than the typical users $\left(\geq 10^{3}\right.$ cells $\left./ \mathrm{km}^{2}\right)$ [18]. It is required state of the art techniques to provide higher spectral efficiency, lower power consumption and densified wireless connections, etc. In my research, I analyzed the performance metrics of average harvested energy on each tier of user, the average ergodic rate (nats/bit/Hz) and the outage probability of rate on UL based on the 2-D cellular network models base on the stochastic geometry HPPP.

A new 3-D model like Fig.21 was proposed for HetNet that the positions of the small cells are distributed by 3-D Poisson point process [19]. I want to make a 2-tier users tiers system that consists of picocells, tier 1 users (smart phone users or pads) and tier 2 users (wearable devices or machine) distributed by HPPP with independent density of each tier users. My purpose is by considering the interference from a 3D HetNet to analysis the power consumption and coverage probability of in 3D HetNet high-rise buildings. 


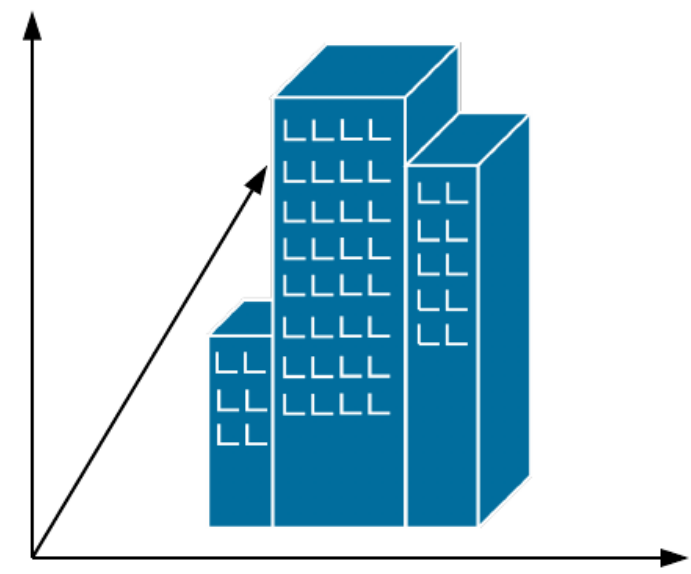

Fig. 21. The 3-D system model based on HPPP 


\section{Appendix}

\section{A proof of Theorem 1}

Based on the Campbell's theorem, we can derive the $\mathbb{E}\left\{I_{B S}\right\}, \mathbb{E}\left\{I_{m u}\right\}, \mathbb{E}\left\{I_{B S}^{\prime}\right\}$ and $\mathbb{E}\left\{I_{m u}^{\prime}\right\}$ as below

$$
\begin{aligned}
& \mathbb{E}\left\{I_{B S}\right\}=\sum_{i \in \Phi_{B S} \backslash\{o\}} P_{B S} g_{b, o} L_{0}\left(\max \left\{\left|X_{i}\right|, d\right\}\right) \\
& =\lambda_{B S} P_{B S} g_{b, o} \beta \int_{0}^{2 \pi} \int_{x}^{\infty}(\max \{r, d\})^{-\alpha_{B}} r d r d \theta \\
& =2 \pi \lambda_{B S} P_{B S} g_{b, o} \beta \int_{x}^{\infty}(\max \{r, d\})^{-\alpha_{B}} r d r \\
& =2 \pi \lambda_{B S} P_{B S} \beta\left(1(x \leq d)\left(d^{-\alpha_{B}} \frac{d^{2}-x^{2}}{2}-\frac{d^{2-\alpha_{B}}}{2-\alpha_{B}}\right)\right. \\
& \left.-1(x>d) \frac{x^{2-\alpha_{B}}}{2-\alpha_{B}}\right) \\
& \mathbb{E}\left\{I_{m u}\right\}=\sum P_{U m} g_{m, o} L_{0}\left(\max \left\{\left|X_{j}\right|, d\right\}\right) \\
& =\lambda_{B S} P_{U m} g_{m, o} \beta \int_{0}^{2 \pi} \int_{0}^{\infty}(\max \{z, d\})^{-\alpha_{U}} z d z d \theta \\
& =2 \pi \lambda_{B S} P_{U m} \beta\left(\frac{d^{2-\alpha_{U}}}{2}+\frac{d^{2-\alpha_{U}}}{\alpha_{U}-2}\right) \\
& \mathbb{E}\left\{I_{B S}^{\prime}\right\}=\sum P_{B S} h_{b, o} L_{0}\left(\max \left\{\left|X_{k}\right|, d\right\}\right) \\
& =\lambda_{B S} P_{B S} h_{b, o} \beta \int_{0}^{2 \pi} \int_{0}^{\infty}(\max \{z, d\})^{-\alpha_{U}} z d z d \theta \\
& =2 \pi \lambda_{B S} P_{B S} h_{b, o} \beta \int_{0}^{\infty}(\max \{v, d\})^{-\alpha_{B}} v d v \\
& =2 \pi \lambda_{B S} P_{B S} \beta\left(\frac{d^{2-\alpha_{B}}}{2}+\frac{d^{2-\alpha_{B}}}{\alpha_{B}-2}\right)
\end{aligned}
$$




$$
\begin{aligned}
\mathbb{E}\left\{I_{m u}^{\prime}\right\}= & \sum_{s \in \Phi_{U m} \backslash\{o\}} P_{U m} h_{m, o} L_{0}\left(\max \left\{\left|X_{S}\right|, d\right\}\right) \\
= & \lambda_{B S} P_{U m} h_{m, o} \beta \int_{0}^{2 \pi} \int_{y^{\prime}}^{\infty}(\max \{u, d\})^{-\alpha_{U}} u d u d \theta \\
= & 2 \pi \lambda_{B S} P_{U m} h_{m, o} \beta \int_{y^{\prime}}^{\infty}(\max \{u, d\})^{-\alpha_{U}} u d u \\
= & 2 \pi \lambda_{B S} P_{U m} \beta\left(1\left(y^{\prime} \leq d\right)\left(\frac{1}{2} d^{-\alpha_{U}}\left(d^{2}-y^{\prime 2}\right)-\frac{d^{2-\alpha_{U}}}{2-\alpha_{U}}\right)\right. \\
& \left.-1\left(y^{\prime}>d\right) \frac{y^{\prime 2-\alpha_{U}}}{2-\alpha_{U}}\right)
\end{aligned}
$$

\section{A proof of Theorem 2}

Theorem 2. The average ergodic rate received at tier 1 user in situation of tier 1 users are full-duplex relay is given by

$$
\begin{aligned}
R_{U m}^{\mathrm{Exact}} & =\mathbb{E}_{\mathrm{SINR}_{U m}}\left\{(1-\tau) \operatorname{Tln}\left(1+\operatorname{SINR}_{U m}\right)\right\} \\
& =\int_{R_{1}>0}^{\infty} \operatorname{Pr}\left((1-\tau) T S I N R_{U m}>R_{1}\right) d R_{1} \\
& =\int_{R_{1}>0}^{\infty} \operatorname{Pr}\left(\operatorname{SIN}_{U m}>e^{\frac{R_{1}}{(1-\tau) T}}-1\right) d R_{1} \\
& =\int_{R_{1}>0}^{\infty} \operatorname{Pr}\left(\operatorname{SIN}_{U m}>t_{1}\right) d R_{1}
\end{aligned}
$$

where base on the Shannon theorem. In addition, the Laplace function generated by the interference from the interfered tier 1 full-duplex relay can be derived as

$$
\begin{aligned}
\mathcal{L}_{I^{\prime} m u}(s) & =\mathbb{E}_{I^{\prime} m u}\left\{\exp \left(-s \sum P_{U m}^{D L} g^{\prime}{ }_{m, o} L_{0}\left(\max \left\{\left|X_{j}^{\prime}\right|, d\right\}\right)\right)\right\} \\
& =\mathbb{E}_{I^{\prime} m u}\left\{\prod \mathbb{E}_{g^{\prime}{ }_{m, o}}\left\{\exp \left(-s P_{U m}^{D L} g^{\prime}{ }_{m, o} L_{0}\left(\max \left\{\left|X_{j}^{\prime}\right|, d\right\}\right)\right)\right\}\right\} \\
& =\mathbb{E}_{I^{\prime} m u}\left\{\prod \mathcal{L}_{g^{\prime}{ }_{m, o}}\left(s P_{U m}^{D L} L_{0}\left(\max \left\{\left|X_{j}^{\prime}\right|, d\right\}\right)\right)\right\}
\end{aligned}
$$




$$
\begin{aligned}
& =\exp \left(-\lambda_{B s} \int_{R^{2}}\left(1-\mathcal{L}_{g^{\prime}{ }_{m, o}}\left(s P_{U m}^{D L} L_{0}\left(\max \left\{\left|X_{j}^{\prime}\right|, d\right\}\right)\right)\right) d x\right) \\
& =\exp \left(-2 \pi \lambda_{B s} \int_{0}^{\infty}\left(1-\mathcal{L}_{g^{\prime}{ }_{m, o}}\left(s \Delta_{U m}(\max \{\varphi, d\})^{-\alpha_{U}}\right)\right) \varphi d \varphi\right) \\
& =\exp \left(-2 \pi \lambda_{B s} \int_{0}^{\infty}\left(1-\frac{1}{1+s \Delta_{U m}(\max \{\varphi, d\})^{-\alpha_{U}}}\right) \varphi d \varphi\right) \\
& =\exp \left(-2 \pi \lambda_{B s}\left(\int_{0}^{d}\left(\frac{s \Delta_{U m} d^{-\alpha_{U}}}{1+s \Delta_{U m} d^{-\alpha_{U}}}\right) \varphi d \varphi\right.\right. \\
& +\int_{d}^{\infty}\left(\frac{s \Delta_{U m} \varphi^{-\alpha_{U}}}{\left.\left.\left.1+s \Delta_{U m} \varphi^{-\alpha_{U}}\right) \varphi d \varphi\right)\right)}\right. \\
& =\exp \left(-2 \pi \lambda_{B s}\left(\frac{s \Delta_{U m} d^{-\alpha_{U}}}{1+s \Delta_{U m} d^{-\alpha_{U}}} \frac{1}{2} d^{2}\right.\right. \\
& \left.\left.+\frac{s \Delta_{U m} d^{\left(2-\alpha_{U}\right)}}{\alpha_{U}-2} 2 F 1\left[1, \frac{\alpha_{U}-2}{\alpha_{U}}, 2-\frac{2}{\alpha_{U}},-s \Delta_{U m} d^{-\alpha_{U}}\right]\right)\right)
\end{aligned}
$$

where $\mathbb{E}\left\{\prod_{x \in \Phi} f(x)\right\}=\exp \left(-\lambda \int_{R^{2}}(1-f(x)) d x\right)$ based on the Hypergeometric [17],

$$
\begin{aligned}
& \mathcal{L}_{g_{m, o}^{\prime}}\left(s \Delta_{U m} \beta(\max \{\varphi, d\})^{-\alpha_{U}}\right) \\
& =\int_{0}^{\infty} \exp \left(-s \Delta_{U m} g_{m, o}^{\prime}(\max \{\varphi, d\})^{-\alpha_{U}}\right) \\
& \cdot \exp \left(-g_{m, o}^{\prime}\right) d g_{m, o}^{\prime} \\
& =\int_{0}^{\infty} \exp \left(-\left(1+s \Delta_{U m} g_{m, o}^{\prime}(\max \{\varphi, d\})^{-\alpha_{U}}\right) g_{m, o}^{\prime}\right) d g_{m, o}^{\prime} \\
& =\frac{1}{1+s \Delta_{U m}(\max \{\varphi, d\})^{-\alpha_{U}}}
\end{aligned}
$$

based on $g_{o}, h_{o} \sim \exp (1)$, and

$$
\int_{d}^{\infty}\left(\frac{s \Delta_{U m} \varphi^{-\alpha_{U}}}{1+s \Delta_{U m} \varphi^{-\alpha_{U}}}\right) \varphi d \varphi
$$




$$
\begin{aligned}
& =\int_{d}^{\infty} \frac{\varphi}{1+\frac{1}{s \Delta_{U m} \varphi^{-\alpha_{U}}}} d \varphi \\
& =\int_{\frac{d^{\alpha_{U}}}{s \Delta_{U m}}}^{\infty} \frac{\left(s \Delta_{U m} t\right)^{\frac{1}{\alpha_{U}}}}{1+t} d\left(s \Delta_{U m} t\right)^{\frac{1}{\alpha_{U}}} \\
& =\frac{\left(s \Delta_{U m}\right)^{\frac{2}{\alpha_{U}}}}{\alpha_{U}} \int_{\frac{d^{\alpha_{U}}}{s \Delta_{U m}}}^{\infty} \frac{t^{\left(\frac{2}{\alpha_{U}}-1\right)}}{t+1} d t
\end{aligned}
$$

where $t_{1}=e^{R_{1} /(1-\tau) T}-1, \Delta_{U s}=P_{U s}^{D L} \beta=\frac{E_{U s}}{(1-\tau) T} \beta, \Delta_{U m}=P_{U m}^{D L} \beta=\frac{E_{U m}}{(1-\tau) T} \beta, \mathrm{R}_{1}$ is the throughput threshold, and we can assume

$$
\begin{gathered}
\frac{1}{s \Delta_{U m} \varphi^{-\alpha_{U}}}=t \\
\varphi=\left(s \Delta_{U m} t\right)^{\frac{1}{\alpha_{U}}} \\
\left(s \Delta_{U m} t\right)^{\frac{1}{\alpha_{U}}}>d \\
t>\frac{d^{\alpha_{U}}}{s \Delta_{U m}}
\end{gathered}
$$

then we can get the limitation of the integration.

The Laplace function of the interference generated by the interfered tier 2 WTDs can be denoted as

$$
\begin{aligned}
\mathcal{L}_{I^{\prime} \text { us }}(s) & =\mathbb{E}_{I^{\prime} m u}\left\{\exp \left(-s \sum_{s^{\prime} \in \Phi_{U s} \backslash\{0\}} P_{U s}^{D L} h_{o, b}^{\prime} L_{0}\left(\max \left\{\left|X_{k}^{\prime}\right|, d\right\}\right)\right)\right\} \\
& =\mathbb{E}_{I^{\prime}{ }_{m u}}\left\{\prod \mathbb{E}_{h_{o, b}^{\prime}}\left\{\exp \left(-s P_{U s}^{D L} h_{o, b}^{\prime} L_{0}\left(\max \left\{\left|X_{k}^{\prime}\right|, d\right\}\right)\right)\right\}\right\} \\
& =\mathbb{E}_{I^{\prime}{ }_{m u}}\left\{\prod \mathcal{L}_{h_{o, b}^{\prime}}\left(s P_{U s}^{D L} L_{0}\left(\max \left\{\left|X_{k}^{\prime}\right|, d\right\}\right)\right)\right\}
\end{aligned}
$$




$$
\begin{aligned}
& =\exp \left(-\lambda_{B s} \int_{R^{2}}\left(1-\mathcal{L}_{h_{o, b}^{\prime}}\left(s P_{U s}^{D L} L_{0}\left(\max \left\{\left|X_{k}^{\prime}\right|, d\right\}\right)\right)\right) d x\right) \\
& =\exp \left(-2 \pi \lambda_{B s} \int_{0}^{\infty}\left(1-\mathcal{L}_{h_{o, b}^{\prime}}\left(s \Delta_{U S}(\max \{k, d\})^{-\alpha_{U}}\right)\right) k d k\right) \\
& =\exp \left(-2 \pi \lambda_{B s} \int_{d_{0}}^{\infty}\left(1-\frac{1}{1+s \Delta_{U S}(\max \{k, d\})^{-\alpha_{U}}}\right) k d k\right) \\
& =\exp \left(-2 \pi \lambda_{B s}\left(\int_{d_{0}}^{d}\left(\frac{s \Delta_{U S} d^{-\alpha_{U}}}{1+s \Delta_{U S} d^{-\alpha_{U}}}\right) k d k\right.\right. \\
& \left.\left.+\int_{d}^{\infty}\left(\frac{s \Delta_{U S} k^{-\alpha_{U}}}{1+s \Delta_{U s} k^{-\alpha_{U}}}\right) k d k\right)\right) \\
& =\exp \left(-2 \pi \lambda_{B s}\left(\frac{s \Delta_{U s} d^{-\alpha_{U}}}{1+s \Delta_{U s} d^{-\alpha_{U}}} \frac{1}{2}\left(d^{2}-d_{0}^{2}\right)\right.\right. \\
& \left.\left.+\frac{s \Delta_{U S} d^{\left(2-\alpha_{U}\right)}}{\alpha_{U}-2} 2 F 1\left[1, \frac{\alpha_{U}-2}{\alpha_{U}}, 2-\frac{2}{\alpha_{U}},-s \Delta_{U s} d^{-\alpha_{U}}\right]\right)\right)
\end{aligned}
$$

where $\mathbb{E}\left\{\prod_{x \in \Phi} f(x)\right\}=\exp \left(-\lambda \int_{R^{2}}(1-f(x)) d x\right)$ based on the Hypergeometric [17], in addition

$$
\begin{aligned}
& \mathcal{L}_{h_{o, b}^{\prime}}\left(s \Delta_{U S}(\max \{k, d\})^{-\alpha_{U}}\right) \\
& =\int_{0}^{\infty} \exp \left(-s \Delta_{U S} h_{o, b}^{\prime}(\max \{k, d\})^{-\alpha_{U}}\right) \cdot \exp \left(-h_{o, b}^{\prime}\right) d h_{o, b}^{\prime} \\
& =\int_{0}^{\infty} \exp \left(-\left(1+s \Delta_{U S} h_{o, b}^{\prime}(\max \{k, d\})^{-\alpha_{U}}\right) h_{o, b}^{\prime}\right) d h_{o, b}^{\prime} \\
& =\frac{1}{1+s \Delta_{U S}(\max \{k, d\})^{-\alpha_{U}}}
\end{aligned}
$$

based on $g_{o}, h_{o} \sim \exp (1)$, and

$$
\begin{aligned}
& \int_{d}^{\infty}\left(\frac{s \Delta_{U s} k^{-\alpha_{U}}}{1+s \Delta_{U s} k^{-\alpha_{U}}}\right) k d k \\
& =\int_{d}^{\infty} \frac{k}{1+\frac{1}{s \Delta_{U S} k^{-\alpha_{U}}}} d k
\end{aligned}
$$




$$
\begin{aligned}
& =\int_{\frac{d^{\alpha_{U}}}{s \Delta_{U S}}}^{\infty} \frac{\left(s \Delta_{U s} l\right)^{\frac{1}{\alpha_{U}}}}{1+l} d\left(s \Delta_{U s} l\right)^{\frac{1}{\alpha_{U}}} \\
& =\frac{\left(s \Delta_{U s}\right)^{\frac{2}{\alpha_{U}}}}{\alpha_{U}} \int_{\frac{d^{\alpha_{U}}}{s \Delta_{U s}}}^{\infty} \frac{l^{\left(\frac{2}{\alpha_{U}}-1\right)}}{l+1} d l
\end{aligned}
$$

where $t_{1}=e^{R_{1} /(1-\tau) T}-1, \Delta_{U s}=P_{U S}^{D L} \beta=\frac{E_{U s}}{(1-\tau) T} \beta, \Delta_{U m}=P_{U m}^{D L} \beta=\frac{E_{U m}}{(1-\tau) T} \beta, \mathrm{R}_{1}$ is the throughput threshold, and we can assume

$$
\begin{aligned}
& \frac{1}{s \Delta_{U s} k^{-\alpha_{U}}}=l \\
& k=\left(s \Delta_{U s} l\right)^{\frac{1}{\alpha_{U}}} \\
& \left(s \Delta_{U s} l\right)^{\frac{1}{\alpha_{U}}}>d \\
& l>\frac{d^{\alpha_{U}}}{s \Delta_{U s}}
\end{aligned}
$$




\section{References}

[1]. Pyattaev A, Johnsson K, Andreev S, et al. Communication challenges in highdensity deployments of wearable wireless devices[J]. Wireless Communications IEEE, 2015, 22(1):12-18.

[2]. Wu G, Talwar S, Johnsson K, et al. M2M: From mobile to embedded internet[J]. IEEE Communications Magazine, 2011, 49(4):36-43.

[3]. Zhang X, Jiang H, Zhang L, et al. An Energy-Efficient ASIC for Wireless Body Sensor Networks in Medical Applications.[J]. IEEE Transactions on Biomedical Circuits \& Systems, 2010, 4(1):11-18.

[4]. Lu X, Niyato D, Wang P, et al. Wireless charger networking for mobile devices: fundamentals, standards, and applications[J]. IEEE Wireless Communications, 2015, 22(2):126-135.

[5]. Dhillon H S, Ganti R K, Baccelli F, et al. Modeling and Analysis of K-Tier Downlink Heterogeneous Cellular Networks[J]. IEEE Journal on Selected Areas in Communications, 2012, 30(3):550-560.

[6]. Zhu Y, Wang L, Wong K K, et al. Wireless Power Transfer in Massive MIMOAided HetNets With User Association[J]. IEEE Transactions on Communications, 2016, 64(10):4181-4195.

[7]. Hossain E, Rasti M, Tabassum H, et al. Evolution toward 5G multi-tier cellular wireless networks: An interference management perspective[J]. IEEE Wireless Communications, 2014, 21(3):118-127. 
[8]. Liu D, Wang L, Chen Y, et al. User Association in 5G Networks: A Survey and an Outlook[J]. IEEE Communications Surveys \& Tutorials, 2015, 18(2):1018-1044.

[9]. Zhu Y, Zheng G, Wang L, et al. Content Placement in Cache-Enabled Sub-6 GHz and Millimeter-Wave Multi-antenna Dense Small Cell Networks[J]. IEEE Transactions on Wireless Communications, 2018.

[10]. Tong Z, Haenggi M. Throughput Analysis for Full-Duplex Wireless Networks With Imperfect Self-Interference Cancellation[J]. IEEE Transactions on Communications, 2015, 63(11):4490-4500.

[11]. Lee J, Quek T Q S. Hybrid Full-/Half-Duplex System Analysis in Heterogeneous Wireless Networks[J]. IEEE Transactions on Wireless Communications, 2015, 14(5):2883-2895.

[12]. Lu X, Wang P, Niyato D, et al. Wireless Networks With RF Energy Harvesting: A Contemporary Survey[J]. IEEE Communications Surveys \& Tutorials, 2017, 17(2):757-789.

[13]. Chandrasekhar V, Andrews J G, Muharemovic T, et al. Power control in twotier femtocell networks[J]. IEEE Transactions on Wireless Communications, 2009, $8(8): 4316-4328$.

[14]. Luo S, Rui Z, Teng J L. Optimal Save-Then-Transmit Protocol for Energy Harvesting Wireless Transmitters[J]. IEEE Transactions on Wireless Communications, 2013, 12(3):1196-1207. 
[15]. Novlan T D, Dhillon H S, Andrews J G. Analytical Modeling of Uplink Cellular Networks[J]. IEEE Transactions on Wireless Communications, 2013, 12(6):26692679.

[16]. Andrews J G, Baccelli F, Ganti R K. A Tractable Approach to Coverage and Rate in Cellular Networks[J]. IEEE Transactions on Communications, 2011, 59(11):3122-3134

[17]. Baccelli F, Błlaszczyszyn B. Stochastic Geometry and Wireless Networks: Volume I Theory[J]. Foundations \& Trends ${ }^{\circledR}$ in Networking, 2009, 3(3-4):249-449. [18]. Kamel M, Hamouda W, Youssef A. Ultra-Dense Networks: A Survey[J]. IEEE Communications Surveys \& Tutorials, 2017, 18(4):2522-2545.

[19]. Omri A, Hasna M O. Modelling and performance analysis of 3-D heterogeneous cellular networks[C]// IEEE International Conference on Communications. IEEE, 2016:1-5. 


\section{Research Achievement}

1. Rong Ye, Kang Kang, Zhenni Pan, and Shigeru Shimamoto, "Wearable Devices Downlink and Uplink Transmission in Multi-hop HetNet with Full-Duplex Relay", in ICMWT conference 2018, HongKong, China, June, 2018. 